Mitteilungen der Österreichischen Geographischen Gesellschaft,

152. Jg. (Jahresband), Wien 2010, S. 29-62

\title{
Weltmacht Indien - Realität Oder Chimäre?
}

\author{
Heinz NiSSEL, Wien*
}

\section{INHALT}

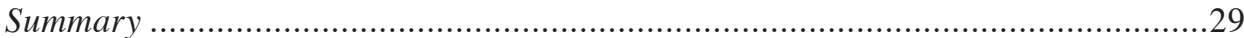

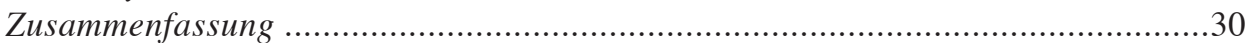

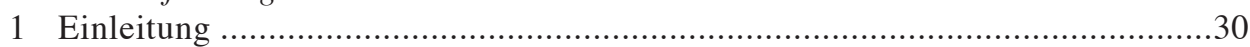

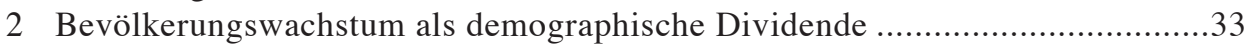

3 Wirtschaftsentwicklung - Trendwende, Aufstieg und Hemmnisse ....................37

4 Stabile Demokratie - fragile Sozialstruktur ..............................................46

5 Außenpolitik, Atommacht und geopolitische Ambitionen ...............................49

6 Das ,,andere“ Indien - Armut, Hunger, Widerstand ........................................53

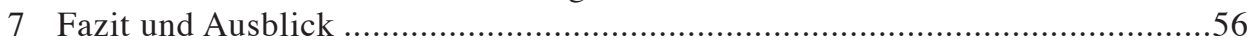

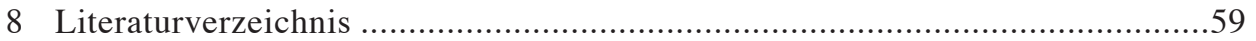

\section{Summary}

Global Power India - Reality or Chimera?

India's rise to global power status has been predicted quite often. This contribution tries to analyse policy guidelines that could either support or contradict such a development. The potential for global power status derives from the size of its population and the demographic dividend of a young age structure, from changes in economic policy and economic growth, from inner stability of the largest democracy on earth, from growing self confidence in foreign affairs, from geopolitical ambitions and military expenditures. By contrast, these developments contribute as well to growing social polarisation, to increasing poverty and hunger, to processes of eviction and resistance. If in the long run India manages to tackle its internal problems and to improve its external relations within South Asia in a peace-building way then the profile of a new policy and role as benevolent global power may take shape.

\footnotetext{
* ao. Univ.-Prof. i.R. Univ.-Doz. Dr. Heinz NISSEL, Institut für Geographie und Regionalforschung, Universität Wien, Universitätsstraße 7,A-1010 Wien; E-mail: heinz.nissel@univie.ac.at, http://www.univie. ac.at/geographie
} 


\section{Zusammenfassung}

Der Aufstieg Indiens zur künftigen Weltmacht ist vielfach prognostiziert worden. Dieser Beitrag versucht Leitlinien zu analysieren, die eine solche Entwicklung bestätigen oder verwerfen könnten. Die potenzielle Weltmacht definiert sich über Bevölkerungs größe und demographische Dividende einer jungen Altersstruktur; Veränderungen der Wirtschaftspolitik und ökonomischen Aufstieg; innere Stabilität der größten Demokratie der Welt; gewachsenes Selbstbewusstsein in der Außenpolitik, in geopolitischen Ambitionen und Aufrüstung. Die Kehrseite wird bestimmt durch Ausweitung sozialer Gegensätze, Armut und Hunger, Verdrängungsprozesse und Widerstand. Wenn es Indien langfristig gelingt, seine internen Probleme zu bereinigen und innerhalb Südasiens friedensstiftend zu wirken, dann ist auch die Profilierung mit einem neuen Politikverständnis in einer globalen Rolle als sanfter Weltmacht denkbar.

"Whatever you can rightly say about India, the opposite is also true." Joan RoBInson

„Die Essenz des indischen Pluralismus besagt, dass man mehrere Dinge zugleich sein kann.“

Shashi THAROOR

\section{Einleitung ${ }^{1)}$}

\subsection{Indien - Selbstbild und Fremdbild}

Über den scheinbar unaufhaltsamen Aufstieg Chinas zur Weltmacht wird in der Öffentlichkeit, von den Medien und von Experten unterschiedlichster Coleur seit Jahrzehnten räsoniert. Lange Zeit weitgehend unbeachtet verharrte hingegen Indien im Windschatten der asiatischen Konkurrenz. Nahezu vergessen war, dass Indien und China schon in vorkolonialer Zeit nicht nur zwei Fünftel der Weltbevölkerung stellten, sondern einen ebenso großen Anteil zum Weltsozialprodukt beisteuerten, und dass der sagenumwobene Reichtum indischer Fürsten überhaupt die Begehrlichkeiten des europäischen Kolonialismus auslöste.2)

\footnotetext{
1) Vor genau 40 Jahren durfte der Autor - damals noch als Student - seinen ersten Aufsatz in den „Mitteilungen“ veröffentlichen: „Ökonomische und soziale Probleme Indiens“ (MÖGG, Band 112, Heft I, 1970, S. 98-119). Die persönliche Faszination durch die überbordende Vielfalt wie die augenscheinliche Unmöglichkeit der Auflösung der Widersprüchlichkeiten des indischen Subkontinents führten zur immer weiteren Beschäftigung mit Fragestellungen in und zu Südasien. In diesen Jahrzehnten durchlief Indien eine kaum vorhergesehene Metamorphose vom Armenhaus zur Regionalmacht mit Ambitionen auf einen künftigen Weltmachtstatus. Der Deutung dieser Transformation - zumindest in einigen wesentlichen Leitlinien - sei dieser „Jubiläumsbeitrag“ gewidmet.

2) Kolumbus entdeckte 1492 auf seinem vermeintlichen Seeweg nach Indien irrtümlich Amerika und dessen Bewohner, die „Indianer“, und 1498 eröffnete VASCO DA GAMA die koloniale Ära mit dem Betreten der Malabarküste Südindiens. Erst im Dezember 1961 wurden die letzten Relikte des Kolonialismus - ironischerweise die portugiesischen Territorien Goa, Daman und Diu - der Indischen Union einverleibt.
} 
Indien als souveräner Staat existiert erst seit 1947, doch in den Augen der Gründungsväter GANDHI und NeHRU und der Intellektuellen schlechthin ist es ein „Immerund-ewig-Land“" (THAROOR 2006), hervorgegangen aus einer 4.000 Jahre alten Zivilisation mit einer gemeinsamen Geschichte und einem gemeinsamen Territorium (von Afghanistan bis Myanmar).

Dieses zu Recht als „Subkontinent“ definierte Gebiet mit seiner unglaublichen Vielschichtigkeit an Sprachen, Religionen und Ethnien ist heute politisch zerstritten und geteilt. Indien als Führungsmacht Südasiens spiegelt diese Komplexität und versteht sich als „Einheit in Vielfalt“, hat aber seinen Nachbarn eine lebendige, pluralistische Demokratie voraus. Indische Vordenker besitzen deshalb häufig ein Gefühl kultureller Überlegenheit und lassen dies neuerdings ihre Gesprächspartner in den großen Verhandlungsrunden (etwa Doha, Kopenhagen, etc.) nur allzu deutlich spüren. Nach Jahrzehnten der internationalen Demütigung und Nichtbeachtung des „Hungerleiders“ oder „Armenhauses“ ist dies eine doch verständliche Reaktion.

Das neue Selbstbewusstsein hat unmittelbare politische Auswirkungen. So zerstörte die Tsunami-Flutwelle vom 26. Dezember 2005 - sie traf vor allem Sumatra - auch zehntausende Leben und Häuser an Indiens Küsten. Doch der Staat lehnte nicht nur jegliche internationale Hilfe $a b$, sondern entsandte seinerseits die Marine zur Notstandshilfe in die Nachbarländer.

\subsection{Licht und Schatten}

Durch Einbinden in die Globalisierung ab 1991 endete eine Phase ökonomischer Isolation und politischer Nichteinmischung. Es folgte ein rasanter wirtschaftlicher Aufschwung mit bahnbrechenden Entwicklungen und dem Aufstieg in die Weltspitze in einigen Sektoren, von denen die Informationstechnologie am bekanntesten ist. Die Mehrzahl der westlichen Experten ist heute überzeugt, dass Indien spätestens 2050 zu den dominierenden Mächten der Welt gehören wird, entweder als gleichwertiger Teil der Triade USA - China - Indien oder knapp hinter den Supermächten (1) China und (2) USA.

Dieser Beitrag möchte die wesentlichen Bestimmungsgründe des Aufstiegs klarlegen. Dabei geht es nicht nur um Veränderungen im Wirtschaftsdenken und dessen Umsetzung in der aktuellen ökonomischen Entwicklung. Eine (mögliche) zukünftige Weltmacht definiert sich auch über Bevölkerungsgröße und demographische Veränderungen, über Kohärenz und Spielräume einer pluralistischen Demokratie im Inneren, über geopolitische Ambitionen durch verstärkte politische Präsenz auf der Weltbühne und militärische Aufrüstung.

Dieser Beitrag möchte aber auch die Kehrseiten des ,anderen“, real existierenden Indien schonungslos aufzeigen - Weltmachtträume konfrontieren mit dem Ist-Zustand, der für die große Mehrheit der Bevölkerung mit bitterer Armut und Hunger einhergeht, mit einem täglichen Überlebenskampf von Millionen Kleinbauern in einer sträflich vernachlässigten Landwirtschaft oder mit Slumbewohnern in den Megastädten, mit grausamen sozialen Ausgrenzungspraktiken (Kastenordnung, Dalits und Adivasi), zunehmender Gewalt, terroristischen Bedrohungen und weiter eskalierender Um- 
weltzerstörung. Langzeitprognosen sind letztendlich so fehleranfällig wie Szenarien zukünftiger Entwicklung. Dem tragen die Titelwahl (mit Fragezeichen) ebenso Rechnung wie die beiden vorangestellten Leitmotive.

\subsection{Vertiefende aktuelle Publikationen}

Dieser Aufsatz versucht Leitlinien zu analysieren, die dem Autor für die aktuelle wie längerfristige Entwicklung Indiens wichtig erscheinen. Es wäre vermessen, diesem Versuch mehr als den Wert einer ersten Einführung in die Problematik zuzuerkennen. Wie im Heft 2/2010 der „Internationalen Politik“ formuliert wird: „Wer Indien verstehen will, muss lesen. Viel lesen“ und „die Neugier, über Indien zu lesen wächst, um doch nur immer neue Fragen aufzuwerfen“ (Jörg Himmelreich). In diesem Sinn seien hier noch aktuelle Literaturzugänge zum Thema nach subjektiver Auswahl mit Anmerkungen des Autors zum Weiterlesen vorgestellt (vgl. auch Literaturverzeichnis).

Während im letzten Jahrzehnt in der anglo-amerikanischen (Fach-)Literatur eine stetig anschwellende Flut von Neuerscheinungen über Indien zu vermerken ist, zeigt der deutschsprachige Raum erst seit wenigen Jahren gesteigertes Interesse. ${ }^{3)}$ Im Jahr 2005 legten Dirk Bronger und Johannes W AMSER einen Vergleich Indien - China aus geographischer Sicht vor. Im Jahr 2006 erschien ein „Weltmacht-Indien“-Schwerpunktheft der Zeitschrift „Internationale Politik“. Im gleichen Jahr oder wenig später erschienen die Bücher von Olaf IHLAU, Bernard IMHASLY, Harald MülleR \& RAUCH, Oliver MüLler und Kurt PILnY, die alle versuchten, der Dynamik der neuen Entwicklungen und der indischen Komplexität gerecht zu werden, aber doch stark die Kernfrage ausklammerten, warum Indien Weltmacht werden soll. Die Autoren sind überwiegend renommierte Journalisten und mehrjährige „residents“ vor Ort. Daraus resultiert auch manch eurozentrische Formulierung.

Tiefer schürfend ist das Buch des Politologen Harald Müller (2. Auflage 2007), gediegen in der Recherche und Analyse das Buch des Nestors der deutschen Indienforschung, Dietmar Rothermund (2008). Recht unterschiedlich präsentiert sich der Sammelband von Michael von Hauff (2009). Für Einsteiger geeignet erweist sich der Überblick von Urs Schoettli (2009).

Michael Mann (2010) legt eine Deutung südasiatischer Geschichte unter indigenem Blickpunkt vor. Erich FriTz (2010) weist Indien die neue Rolle globaler Verantwortung $\mathrm{zu}$. Sehr gelungen ist das soeben erschienene Heft der Edition Le Monde diplomatiQue (Nr. 7, 2010), das die Schattenseiten der Globalisierungseinflüsse betont. Auch Heft 1/2010 der Zeitschrift SüDASIEN enthält lesenswerte Beiträge.

Unter den englischen Veröffentlichungen ragt der rundum gelungene Band von Edward LuCE (2007) heraus. Die datenreiche Wirtschaftsanalyse von Arvind PANAGARIYA (2008) ist „state of the art“. Für den Vergleich Indien - China und die globalen Auswirkungen: WinTERs \& SHAHID 2007.

\footnotetext{
3) Einem breiteren Kreis öffnete sich die schöpferische Vielfalt Indiens in Belletristik und Fachliteratur erst durch die Einladung als Schwerpunkt- und Gastland bei der 58. Internationalen Buchmesse in Frankfurt am Main im Oktober 2006 unter dem Motto „Today“s India“.
} 
Hervorragende Einstiege in die indische Außenpolitik liefern die Bücher von Tobias Engelmeier (2009) und Sumit Ganguly (2010). Außerdem sind die wichtigsten Tageszeitungen und Zeitschriften Indiens allesamt online zugänglich (vgl. Literaturverzeichnis).

\section{Bevölkerungswachstum als demographische Dividende}

\subsection{Das heterogene Bild der Bevölkerungsentwicklung}

Indien befindet sich seit der letzten Volkszählung (2001) mit 1.027 Mio. Einwohnern als zweites Land der Erde in der „Milliardenliga“. Damit leben 17,5\% der Menschheit auf nur 2,4\% der Erdoberfläche, da Indien mit 3.287 Mio. $\mathrm{km}^{2}$ „nur“ das siebentgrößte Land der Erde ist. Mit der kommenden Volkszählung (VZ) im Frühjahr 2011 dürfte die Bevölkerungszahl bei etwa 1,2 Mrd. liegen, und nach Prognosen der $\mathrm{UNO}^{4)}$ könnte sie bis 2050 auf 1,63 Mrd. ansteigen. Etwa zwischen 2025 und 2035 würde Indien dabei die Volksrepublik (VR) China überholen und zum bevölkerungsreichsten Land der Erde werden.

Bisher ergibt sich für die Bevölkerungsentwicklung ein sehr heterogenes Bild. Fortschritte im Kampf gegen Kinder- und Säuglingssterblichkeit, das Zurückdrängen des Analphabetismus und die Reduktion genderspezifischer Ungleichheit der Lebenschancen sind zweifellos vorhanden, aber im Vergleich zu anderen Schwellenländern noch zu gering und zu langsam. Die Geschlechterproportion wird jährlich ungleicher: 2008 entfielen auf 112 Knaben- nur 100 Mädchengeburten. Wegen weit verbreiteter Diskriminierungspraktiken und Abtreibungen weiblicher Föten ,fehlen“ bereits nicht weniger als 35 Mio. Mädchen und Frauen (VZ 2001)! Patriarchalisch organisierte Familien- und Dorfstrukturen, weit verbreitete Armut, Mangel- und Fehlernährung tragen dazu bei. Die Bevorzugung von Söhnen führte trotz Verbots zu einem Boom pränataler Ultraschalluntersuchungen und damit einhergehender Abtreibungen.

Bei der Größe des Landes ergeben sich für alle demographischen Variablen große Spannweiten nach Regionen, nach rural-urbaner Differenzierung, nach Einkommen, Bildung, Religion, Kaste usw. Da in Indien bei Wahlen das (ehemals britische) Mehrheitswahlrecht zur Anwendung kommt, könnte das zunehmende demographische Auseinanderdriften des Nordens und Südens zu einer ernsten Belastung führen.

Indien hat - im Gegensatz zu China - eine lange Tradition der Datensammlung und Aufbereitung mit Vollerhebungen seit bald 150 Jahren, wobei im Jahr 2011 der 15. Zensus stattfinden wird. ${ }^{5}$ Wie bei vielen Problemen des Landes beeindruckt allein schon die schiere Größenordnung der Operation. Über zwei Millionen Erhebungsbeamte werden Fragebogen in 18 unterschiedlichen Sprachen (und Schriften) austeilen und

\footnotetext{
4) UN World Population Prospect. The 2008 Revision Population Database.

5) Nach Registrierung allgemein zugänglich ist die Homepage http://www.censusindia.net oder http://www. censusindia/gov.in. Das Problem liegt nicht im „Data Mining“, sondern in der Überfülle des Angebots und in den Mängeln der Analyse und Aufbereitung durch Ministerien, etc.
} 
bearbeiten. Frühere Volkszählungen ergaben Stagnation oder sogar negative Bilanzen, hervorgerufen durch Hungerepidemien, Massenerkrankungen, Naturkatastrophen usw.

Bei der ersten Volkszählung nach der Unabhängigkeit im Jahr 1951 wurden 361 Mio. Menschen gezählt. Durch bessere Nahrungsversorgung und medizinische Betreuung wuchs die Bevölkerung kontinuierlich zwischen 2,0 und 2,3 Prozentpunkte pro Jahr. In den letzten Jahren sinkt die Zunahmequote, vor allem in Südindien, deutlich ab und bewegt sich im Jahr 2001 bei 1,5\% im Schnitt, 2007 nur noch bei 1,38\%.

Im Modell des demographischen Übergangs wandelt sich Indien heute vom „,verschwenderischen“ (hohe Geburten- und Sterberaten) zum ,sparsamen“ Bevölkerungstyp (niedrige Raten). Da zunächst die Sterberaten sinken und (manchmal erst nach Jahrzehnten) die Geburtenraten ähnlich deutlich nachziehen, kommt es in dieser Übergangsphase zur starken Ausweitung der Population. In nur 50 Jahren hat sich deshalb die Bevölkerung Indiens verdreifacht.

Die Reduktion von Mortalität stößt auf breite gesellschaftliche Akzeptanz, ungleich schwieriger ist die Senkung von Fruchtbarkeits- und Geburtenraten. Es gilt, uralte religiöse Einstellungen und soziale Praktiken zu ändern, über bessere ökonomische Rahmenbedingungen sowie Akzeptanz von Bildung, und besonders über verbesserte Chancen für Mädchen. Früher musste ein Ehepaar (auch ähnlich in China) drei Söhne „produzieren“ (bedeutet statistisch zusätzlich drei Töchter), um zweckrational die eigene Altersversorgung abzusichern.

\subsection{Verbesserte Lebensbedingungen, aber verstärkter Nord-Süd-Gegensatz}

Die verbesserten Lebensbedingungen wirken sich heute bereits deutlich im demographischen Verlauf aus. Im generativen Verhalten besitzen Städter einen Innovationsvorsprung, doch sinken - wenn auch bei höherem Ausgangsniveau - die Geburtenraten im ländlichen Raum gleich schnell. Ähnliche Trends zeigen sich bei der Kinder- und Säuglingssterblichkeit. Im Jahr 2008 lag die Geburtenrate bei 22,2 Promille, die Sterberate bei 6,4 Promille, die Fruchtbarkeitsrate bei 2,76. Dramatisch verbessert hat sich als Ergebnis dieser Trends die mittlere Lebenserwartung. Im Jahr 1930 lag sie bei nur 29 Lebensjahren, beim Zensus 2001 bei 62; inzwischen liegt sie schon bei 69,2 (Männer 66,9; Frauen 71,9). Innerhalb von nur 80 Jahren hat sich die durchschnittliche Lebensspanne damit weit mehr als verdoppelt! Zwischen 1991 und 2001 wuchs die Bevölkerung dabei um nicht weniger als 181 Mio. Menschen. Damit übertraf die Zunahme allein im letzten Jahrzehnt deutlich die fünftgrößte Population weltweit, Brasilien, und noch immer gilt die Aussage Indira GANDHIs aus der Mitte der 1970er Jahre: „We add an Australia every year.“ Nur vor diesem Hintergrund wird deutlich, warum Indien bisher nicht in der Lage war, die entsprechende soziale Infrastruktur für seine Bewohner zu schaffen. Aber welcher (auch wohlhabendere) Staat wäre dazu in der Lage gewesen?

Zu den großen demographischen Herausforderungen zählt auch eine bei der Größe des Landes nicht verwunderliche, sehr unterschiedliche Entwicklung in den einzelnen Bundesstaaten und Territorien, vor allem ein deutlicher Nord-Süd-Gegensatz mit möglichen weitreichenden politischen Konsequenzen. Der Geburtenrückgang ist viel 
stärker in den südlichen Teilen der Union ausgeprägt als im „Hindi belt“ von Bihar, Uttar Pradesh, Madhya Pradesh und Rajasthan. Dies spiegelt die unterschiedlichen ökonomischen Erfolge und die unterschiedliche Mobilisierung von Humanressourcen (u.a. Alphabetisierungskampagnen, Einschulungen etc.) wider. Indiens ärmste Region, das krisengeschüttelte Bihar, weist zwischen 1991 und 2001 einen Bevölkerungszuwachs von $28,4 \%$ auf, das südindische Kerala nur (noch) von 9,4\%.

Politisch gesehen kann diese ungleiche Entwicklung die „größte Demokratie der Welt“ über kurz oder lang vor eine Zerreißprobe stellen. Denn nach dem Mehrheitswahlrecht wurden die Parlamentssitze des jungen Staates proportional nach der anfänglichen Bevölkerungsverteilung vergeben. Im Jahr 2000, noch unter der damaligen Regierung der Bharatiya Janata Party (BJP) unter Premier VAJPAYEE, erfolgte ein Einfrieren der regionalen Sitzverteilung auf 25 Jahre - da sonst die erfolgreicheren Bundesstaaten für ihre sozioökonomischen Fortschritte politisch bestraft worden wären. Damit verschiebt sich aber die Stimmgewichtung immer mehr. Kostet zum Beispiel im Jahr 2025 ein Grundmandat in Bihar dreimal so viele Wählerstimmen wie in Kerala, wäre der Sinn des One-man-one-vote-Prinzips in Frage gestellt. Eine weitere Gefahr liegt im beständigen Aufwind von Parteien (nur) regionalen Zuschnitts, die gleichwohl für die Regierungsbildung unentbehrlich geworden sind.

\subsection{Einschätzung des Wachstums - vom Malus zum Bonus}

Indien sollte nicht nur geopolitisch, sondern auch bevölkerungspolitisch nicht als Inselstaat, sondern als Kernraum und Führungsmacht Südasiens gesehen werden. Bezieht man die mehr als 350 Mio. Einwohner von Pakistan, Bangladesch, Nepal und Sri Lanka mit ein, ergeben sich 1,5 Mrd. Bewohner, also bereits mehr als die der beiden großen Flächenstaaten Asiens, China und Russland, gemeinsam! Im Jahr 2050 stünden dann 2,2 Mrd. Südasiaten 1,4 Mrd. Chinesen gegenüber (unter Beibehaltung der dortigen Ein-Kind-Politik).

Jahrzehntelang hielt man auf dem Subkontinent die Bevölkerungs"explosion" für die größte Katastrophe und das entscheidende Hindernis für jegliche (ökonomische) Entwicklung. In den 1960er und 1970er Jahren räumte Indien deshalb der Familienplanung höchsten Stellenwert ein und Sanjai GANDHI, der ältere Sohn Indiras, versuchte mit brutalen Zwangsmaßnahmen die Zwei-Kind-Familie durchzusetzen. (Slogan „Four is a family, five is a crowd.“) Obwohl diese Programme letztendlich am Widerstand der Bevölkerung scheiterten, wurden die Behörden doch nicht müde zu betonen, wie viele Millionen Geburten dadurch eingespart werden konnten - zum Wohl der Gesellschaft und zur Verbesserung der ökonomischen Rahmenbedingungen.

Interessanterweise hat sich die Einschätzung heute völlig in das Gegenteil verkehrt. Experten erklären nun, dass der größte demographische Vorteil Indiens in Zukunft gerade darin bestünde, dass die Alterspyramide im Vergleich zu China, Europa und Russland bis 2050 eine ,junge“ bleibt. Zurzeit gehören 31,5\% zu den bis 14-Jährigen, $63,3 \%$ zur ,produktiven Gruppe“ der 15- bis 64-Jährigen und nur 5,2\% sind älter (65+). Da China deutlich früher die Phasen des demographischen Übergangs durchlaufen hat, eröffnen sich für Indien noch auf Jahrzehnte hinaus Vorteile, die „demographischer 
Bonus“ oder „demographische Dividende“ genannt werden (Lutz \& SCHERbov 2005). Während Gesamtbevölkerungswachstum einen (statistisch signifikant) negativen Effekt auf das Pro-Kopf-Einkommen hat, wirkt sich umgekehrt das Anwachsen der ökonomisch aktiven Bevölkerung signifikant positiv aus. China weist im Jahr 2008 einen Spitzenwert von 71\% erwerbsfähiger Bevölkerung an der Gesamtpopulation aus. In Indien wird dies erst um 2030 der Fall sein. Die kommenden Jahrzehnte bedeuten für Indien ein einzigartiges „window of opportunity“ hinsichtlich altersstruktureller Vorteile für wirtschaftliches Wachstum und erhöhte Produktivität. Dafür ist allerdings ein kompletter Mix aus politischen, ökonomischen und demographischen Strategien notwendig (Kugler \& Swaminathan 2006).

\subsection{Alphabetisierung und Urbanisierung}

Von vorrangiger Bedeutung für die Ausschöpfung dieses Potenzials sind die energische Bekämpfung des Analphabetismus, die Verbesserung des (Primär-)Schulwesens und der Bildungs- und Berufschancen der Mädchen. In der Alphabetisierung konnten in der letzten Dekade deutliche Fortschritte verzeichnet werden. Sie sind jedoch erheblich geringer als in China. Rund zwei Drittel aller Bewohner (über sechs Lebensjahren) können heute Lesen und Schreiben, aber noch immer mit einer deutlichen „gender bias“ - ,nur“" ein Viertel der Männer sind Analphabeten, jedoch die Hälfte der Frauen. Die Schere zwischen den Geschlechtern schließt sich, d.h. die Mobilisierung der Grundbildung für Frauen beginnt endlich zu greifen. In fortschrittlichen Bundesstaaten liegt der Mobilisierungsgrad bereits über 90\%. Die absolute Zahl der Analphabeten beginnt aber erst seit einigen Jahren zu sinken. Trotz vieler Einschränkungen müssen die Fortschritte der Bildungsoffensive gewürdigt werden. Dies zeigt ein Vergleich mit dem Bildungsniveau von 1951 - damals konnte gerade ein Viertel der Männer und nicht einmal jede zehnte Frau lesen und schreiben.

Verstärkte Aufmerksamkeit muss in Zukunft auch der Urbanisierung gelten. Mit rund 300 Mio. Stadtbewohnern wird das urbane Potenzial Indiens quantitativ nur von China übertroffen. Doch waren dies im Jahr 2001 weniger als 28\% der Gesamtbevölkerung. Damit zählt die Verstädterungsquote Indiens zu den niedrigsten weltweit. Auch das Tempo der Urbanisierung gehört zu den schwächsten und hat sich innerhalb der letzten 30 Jahre weiterhin verlangsamt! Andere Staaten Asiens mit ursprünglich niedrigerem Ausgangsniveau wie China, Thailand oder Indonesien haben Indien inzwischen deutlich überholt

Die Gründe dafür sind vielfältig, sollen aber an dieser Stelle nicht weiter verfolgt werden (vgl. dazu Nissel 2006, 2007). Noch hat der Zuzug in die Großstädte aus den 640.000 Dörfern nicht in vollem Ausmaß eingesetzt. Die Lebensumstände in Indiens Metropolen gehören aber schon jetzt zu den schlimmsten weltweit.

Trotz niedriger Urbanisierung spielen die Metropolen und Megastädte in der Wirtschafts- und Sozialentwicklung des Landes eine herausragende Rolle (NISSEL 2009). Die New Economic Policy (NEP) steuert über sie die Globalisierungseinflüsse auf Ökonomie und Bevölkerung. Die Megacities Bombay [Mumbai], Delhi und Kalkutta [Calcutta/Kolkata] zählen zu den zehn größten Stadtagglomerationen der Erde. Bei 
anhaltenden Trends dürfte zwischen den Jahren 2020 und 2025 Greater Mumbai als neue Nummer eins Tokio [Tōkyō] in der Weltstädtehierarchie ablösen.

Die Lösung (auch) der demographischen Probleme ist eine wesentliche Voraussetzung für die Verwirklichung politischer und ökonomischer Großmachtträume. Der Bewältigung der Armuts- und Hungerproblematik kommt dabei eine entscheidende Bedeutung zu (vgl. Kapitel 6).

\section{Wirtschaftsentwicklung - Trendwende, Aufstieg und Hemmnisse}

\subsection{Von der Planwirtschaft zur New Economic Policy (NEP)}

Durch vier Jahrzehnte, zwischen 1951 und 1991, entwickelte sich die indische Wirtschaft trotz oder wegen weitreichender staatlicher Steuerung nur langsam. Die Wirtschaftsstrategie orientierte sich am Binnenmarkt (Importsubstitution), teils vom Kampf um die Unabhängigkeit geprägt („swadeshi“-Bewegung/Autarkiestreben), teils inspiriert vom Aufbau der Sowjetunion. Nach deren Vorbild erstellte die wie eine Nebenregierung agierende allmächtige Planungskommission unter Vorsitz des Premiers Jawaharlal NeHRU Fünfjahrespläne, wobei im Rahmen einer „mixed economy“ alle Schlüsselbereiche in der Hand des Staates verblieben. Genau so verfolgte seine Tochter Indira GANDHI während ihrer Regierungszeiten stetig ihre Vision vom „right way towards a socialist pattern of society“. Dies passte gut zur politischen Strategie, in der sich Indien als Weltführer einer von der Tendenz her antikolonialistischen oder antiwestlich orientierten Bewegung der Blockfreien (Länder) positionierte (Achse NeHRU-Tito-NASSER).

Das mit dieser Führungsrolle erworbene außenpolitische Ansehen wurde aber durch die zentralistische Gängelung der Wirtschaft konterkariert. Das Wachstum beschränkte sich bis in die 1980er Jahre auf ca. 3,5\% im Jahr, für die der Ökonom Raj KRISHNA die ironische Formel von der „Hindu rate of growth“ prägte. Bei einem Bevölkerungswachstum von 2 bis 2,3\% per annum blieb kaum ein Wachstumsimpuls für die Verbesserung der Lebensbedingungen übrig. RоTHERMUnD vertritt allerdings die interessante These, dass gerade diese Langsamkeit des Wirtschaftswachstums den sozialen Frieden bewahrte. Zumindest brachten die Erfolge der „Green Revolution“ 1982 - wahrscheinlich erstmals in der Jahrtausende umspannenden Geschichte - die Selbstversorgung mit Lebensmitteln. Die Wirtschaft Chinas hingegen wuchs schon damals und immer weiter um jährlich zehn und mehr Prozentpunkte. Und dies, obwohl in den 1960er Jahren Indien durchwegs noch bessere ökonomische Indikatoren aufwies als China - nicht zuletzt dem britischen Kolonialerbe geschuldet (BRONGER \& WAMSER 2005).

Im Jahr 1991 erfolgte dann recht plötzlich die völlige Kehrtwende in der indischen Wirtschaftspolitik. Bedingt durch den ersten Golfkrieg und die davon ausgelöste Ölknappheit, wegen einer schweren Zahlungsbilanzkrise und Devisenreserven, die nur noch für wenige Wochen reichten, geriet Indien in eine schwere Finanz-, ja Staatskrise. Premier Narasimha RaO und sein Finanzminister Manmohan SINGH (seit 2004 Pre- 
mier, 2009 wiedergewählt) vermochten sich aus dieser Lage nur durch einen radikalen Paradigmenwechsel zu Gunsten einer Liberalisierung von Handel und Kapitalverkehr zu retten. SINGH gilt damit als Vater der New Economic Policy (NEP), die einen neoliberal-marktwirtschaftlichen Kurs fährt und damit Indien für Globalisierungseinflüsse geöffnet hat. Seit 1992/93 läuft Indien auf der ökonomischen Erfolgsschiene. Das lähmende Lizenzsystem ist weitgehend verschwunden, die Privatisierung staatlicher Monopole wird vorangetrieben, die Zölle wurden um ein Vielfaches verringert, Handelshemmnisse abgebaut, und Schritt für Schritt ausländische Kapitalmehrheiten in immer mehr Industrie- und Dienstleistungsbranchen erlaubt. (In einigen Branchen wie der Pharma-, der Autoindustrie sowie der Telekommunikation sind ausländische Beteiligungen bis zu $100 \%$ genehmigt.)

Im indischen Kontext kommen Deregulierung und Privatisierung einem Erdrutsch gleich. Doch im Vergleich zu anderen Ländern gibt Indien durchaus nicht alle Kontrollen aus der Hand; so etwa nicht im Finanzsektor, worauf Experten die relativ geringen Auswirkungen der Weltfinanz- und Wirtschaftskrise seit Herbst 2008 auf Indien zurückführen.

\subsection{Wachstum des Bruttoinlandsprodukts (BIP)}

In den 1990er Jahren erzielte Indien daraufhin bereits 6\% Wachstum pro Jahr. Zwischen 1986 und 2006 verdreifachte sich das Bruttoinlandsprodukt (MüLLER \& RAUCH 2008). Im Fiskaljahr 2003/04 (April bis März) stieg es um 8,5\%, 2004/05 um 7,5\%, 2005/06 um 8,1\%,2006/07 um 9,0\%, 2007/08 um 9,1\% - diese Größenordnung wird nur von der VR China übertroffen.

Es sind diese enormen Wachstumssteigerungen des BIP, welche die Phantasien inund ausländischer Beobachter beflügelten und die daraufhin aus dem ewigen Armenhaus gleich eine kommende Supermacht wachsen sehen wollten. Spätestens ab 2010/11 wollte die indische Regierung die jährliche Wachstumsrate auf zehn und mehr Prozent anheben. Dies ist aufgrund der indirekten Auswirkungen der Weltfinanzkrise unmöglich geworden. Indien hat sie zwar besser überstanden als die Erste Welt, jedoch nicht so gut wie China. Ein Vergleich: Im ersten Quartal 2008 lag das BIP-Wachstum Indiens noch bei 8,5\%, im zweiten Quartal bei 7,6\% (China 10,1\%), im dritten Quartal bei 7,5\% (China 9,0\%). Im vierten Quartal 2008 kam der deutliche Abschwung - Indien 6,2\%, China 6,8\%. Im ersten Quartal 2009 wurde die Talsohle erreicht: Indien 5,8\%, China 6,2\%. Seither geht es wieder bergauf - zweites Quartal 2009: Indien 6,1\%, China 7,9\%; drittes Quartal 2009: Indien 7,9\%, China 9,1\%; viertes Quartal 2009: Indien fällt auf $6,0 \%$ zurück, China ist mit $10,7 \%$ wieder im zweistelligen Bereich und setzt dies im ersten Halbjahr 2010 fort (noch keine Daten für Indien) (Quelle: ASIENKURIER Nr. 38, 7/20106).

\footnotetext{
6) Der AsIenkurier ist eine seit Juli 2007 monatlich erscheinende, frei zugängliche online-Zeitschrift. Sie enthält aktuelle Beiträge zur Ökonomie asiatischer Staaten und fortlaufend revidierte Tabellen wichtiger Wirtschaftsdaten - http://pdf.asienkurier.com.
} 
Die Performanz Indiens ist im internationalen Vergleich trotzdem ausgezeichnet, obwohl führende Ökonomen die Auffassung vertreten, dass Indien für einen kontinuierlichen Aufschwung zumindest $8 \%$ BIP-Wachstum im Jahr benötigt. Sie schätzen aber auch, dass das BIP-Wachstum um bis zu zwei Prozentpunkte im Jahr höher sein könnte, wenn die Infrastruktur nicht so schlecht wäre.

Noch immer leben 26\% der Einwohner unterhalb der (indischen) Armutsgrenze, doch waren dies am Ende der Planwirtschaft im Jahr 1991 noch 43\%. Die Realeinkommen (nach Kaufkraftparität) haben sich in dieser Zeit verdoppelt. Das verfügbare Pro-Kopf-Einkommen ist mit 820 Dollar pro Jahr (2007) jedoch noch recht bescheiden. $\mathrm{Ob}$ dieses Wachstum struktureller oder (nur) zyklischer Natur ist, bleibt umstritten. Nach wie vor beurteilen aber die wichtigsten Finanzinstitutionen - Weltbank, Internationaler Währungsfonds (IWF), Asiatische Entwicklungsbank - die ökonomische Entwicklung Indiens sehr positiv, was unter anderem ein rapides Ansteigen der ausländischen Direktinvestitionen in den letzten Jahren zur Folge hatte.

Folgt man der berühmt gewordenen und häufig zitierten Studie von Goldman \& SACHS (2003), dann dürfte das Bruttosozialprodukt künftig jenes der folgenden Staaten in den folgenden Jahren übertreffen: Italien im Jahr 2014, Frankreich 2020, Deutschland 2023 und Japan 2033 (damit an dritter Stelle hinter China und den USA).

\subsection{Neue Mittelschichten, Konsum und Privatwirtschaft}

Diese insgesamt positive Entwicklung ist im Einzelnen mit vielen Nachteilen erkauft. Sie beruht überwiegend auf dem Konsumdrang der „neuen Mittelschicht(en)“ und massiven ausländischen Kapitalzuflüssen in indische Aktien (Gosh 2010). Es wäre jedoch verfehlt, die Wirtschaftsentwicklung nur über das BIP zu definieren. Zugleich existiert ein massiver Kaufkraftverlust - bis Februar 2010 verdoppelte sich die Inflationsrate auf $10 \%$ innerhalb eines Jahres. Die Lebensmittelpreise stiegen sogar um 18\%. Die häufig so apostrophierte „,neue Mittelschicht“ soll angeblich bereits 300 und mehr Millionen Menschen umfassen. Dem muss aber widersprochen werden: Es sind 50 bis maximal 150 Mio. - und auch die nur bezogen auf das indische Einkommensspektrum. Rund 75 Mio. Haushalte (nicht Einzelpersonen!) verfügen über ein Jahreseinkommen zwischen 1.000 und 4.000 Euro, weitere 40 Mio. über ein solches zwischen 4.000 und 8.000 Euro. Angehörige dieser Einkommensgruppen tragen überwiegend den Wirtschaftsaufschwung mit ihrer Konsumfreudigkeit. (Der Nano, das neue „Wunderauto“ der Tata-Gruppe, kam im Jahr 2009 für 1.700 Euro auf den Markt.) Nur 1,2 Mio. Haushalte kommen auf mehr als 8.000 Euro.

Nicht wenige Inder sind heute sehr stolz darauf, dass das Land zwei Dutzend Dollarmilliardäre besitzt, vier der zehn reichsten Männer der Welt und dass der Selfmademan und Stahlmagnat Lakshmi MitTal zu den drei reichsten Menschen zählt. Im Jahr 2010 soll der Konsumgütermarkt bereits zu den fünf größten der Welt gehören. Zu den wichtigen Komponenten zählen zunehmende Motorisierung, Anschaffung von Haushaltsgeräten und der Massentourismus. Das so hoch gelobte Zugpferd der indischen Wirtschaft, die Informationstechnologie(IT)-Branche, kann zur dringend benötigten Schaffung von Millionen Arbeitsplätzen (und das jährlich!) nur sehr wenig beitragen. 
Indiens Privatwirtschaft - auch hier wieder in deutlichem Gegensatz zu China - zeichnet sich durch die Dominanz großer Dynastien aus. So besteht etwa der TataKonzern aus einem Konglomerat von an die 100 Tochtergesellschaften, darunter Stahlproduzent, Autobauer, Chemie-Riese, Betreiber der größten Luxushotelkette ursprünglich Gründer und Betreiber der Air India - Großeigner und Vermarkter von Teeplantagen bis hin zur Consultingagentur für schlüsselfertige industrielle Gesamtlösungen mit Office in New York. Auch Namen wie BiRLA, Godrej oder Mittal kennt in Indien jedes Kind. Diese Konzerne können die besten Arbeitssuchenden auswählen und verfügen deshalb über loyale, hoch motivierte Mitarbeiter. Auch kleinere Firmen der „creative industries“ können sich auf dem Weltmarkt nicht nur behaupten, sondern auch kräftig expandieren (Softwareentwickler, Textilerzeuger, Modedesigner, Juweliere, Filmindustrie á la „Bollywood“ usw.)

\subsection{Boomregionen und der, ,Rest““}

Wie in der Bevölkerungsentwicklung existieren auch bezüglich der ökonomischen Veränderungen enorme regionale Unterschiede. Ausgesprochene Boomregionen sind die Bundesstaaten im Westen, Maharashtra und Gujarat, dann im Norden die „National Capital Region“ von Delhi mit den neuen Wachstumsmotoren von Noida, Gurgaon und Faridabad, die Bundesstaaten Punjab und Haryana sowie der Süden, vor allem Tamil Nadu und Karnataka. Die größten Wachstumsimpulse gehen jedoch von den Megastädten aus (Ahuja \& Brosius 2006, Nissel 2006, 2009), unangefochten in erster Linie von der „Wirtschaftshauptstadt“ Bombay, zunehmend von Delhi und Bengaluru, aber auch von den konkurrierenden Landeshauptstädten des Südens, Madras [Chennai] und Haidarabad [Hyderabad]. Westbengalen mit Kalkutta liegt deutlich zurück, hat aber in den letzten Jahren eine Aufholjagd gestartet. Der „Rest“ Indiens, die ärmeren Bundesstaaten im Norden, Nordosten und im zentralen Dekkan sowie viele Inselregionen überall im Land werden von Jahr zu Jahr stärker von der Wirtschaftsentwicklung abgekoppelt.

Sowohl staatliche wie private, in- wie ausländische Akteure bevorzugen in ihrem Investitionsverhalten die Megacities und zum Teil noch andere Metropolen bei gleichzeitiger Vernachlässigung des ländlichen Raumes und der Städte niedrigerer hierarchischer Kategorien. Die New Economy benötigt für die Durchsetzung ihrer Ziele qualifiziertes Humankapital und den Einsatz innovativer Hochtechnologie, beides verankert in den wichtigen städtischen Agglomerationen. Aufgrund der stark gestiegenen Lohnkosten (pro Jahr 20-30\%) diffundiert die InformationstechnologieIndustrie erst in jüngster Zeit in kleinere Sekundärzentren.

Seit 1956 strebte der Staat in Phasen der Planwirtschaft nach regionalem Ausgleich der Entwicklung. Es war dies das zweite, gleichwertige Oberziel neben der Stärkung der Wirtschaftsleistung. Die Maximen der NEP verlangen hingegen eine Konzentration auf die „Anker“ der globalen Ökonomie. Seit 1991 hat das Prinzip des Gleichklangs von Wirtschafts- und Regionalpolitik ausgedient, heute herrscht der Primat ersterer. Wie noch zu zeigen sein wird, breiten sich die wachsenden Disparitäten von Lebens- und Entwicklungschancen nicht ungestraft aus, sondern fordern einen hohen politischen Preis. 


\subsection{Außenhandel und Investitionen}

Im Vergleich zu asiatischen Konkurrenten erscheint der indische Außenhandel noch immer unterentwickelt. Er beträgt nur etwa ein Viertel des BIP, die Exporte machen gar nur ein Zehntel aus. Dies liegt zum einen an der langen bewussten Abschottung nach dem Prinzip der „,self reliance“, zum anderen an der lange nicht gegebenen Konkurrenzfähigkeit indischer Produkte. Auch dies ändert sich aber sehr rasch. Im Jahr 1986 lagen die Exporte erst bei 10,4 Mrd. Dollar, 2005/06 exportierte Indien erstmals Waren im Wert von mehr als 100 Mrd. Dollar (ein Zuwachs um 25\% gegenüber dem Vorjahr). Allerdings stiegen die Importe noch stärker, nämlich auf 140 Mrd. Dollar (+32\%). Ein Drittel davon musste allein für Ölimporte gezahlt werden. Die Industrieexporte wuchsen von 37 Mrd. (2002/03) auf 85 Mrd. Dollar (2005/06). Im gleichen Fiskaljahr erreichten die Dienstleistungsexporte inklusive Software bereits 23,6 Mrd. Dollar, im Jahr 2009 rund 50 Mrd. und damit ein Drittel der Gesamtexporte. Die Boomdekade mit 20 und mehr Prozent Wachstum pro Jahr ist jedoch vorbei. Für 2010 werden nur noch $5,5 \%$ erwartet.

Die Beratungsfirma McKinsey schätzt die Ausweitung indischer Exporte bis zum Jahr 2015 auf 300 Mrd. Dollar und prognostiziert einen Weltmarktanteil Indiens von $3,5 \%$. Dabei ist anzumerken, dass der Handelsaustausch mit den Nachbarstaaten bisher nur gering ist (nur 4\% der Exporte), trotz der Bemühungen um intensivere Handelsbeziehungen im Rahmen der South Asian Association for Regional Development (SAARC), einem Pendant zur Association of Southeast Asian Nations (ASEAN). Zu groß scheinen nach wie vor die wechselseitigen Animositäten, die politischen und militärischen Friktionen zu sein. Auch bei den ausländischen Direktinvestitionen lag Indien über die Jahrzehnte hoffnungslos hinter China zurück (mit Summen um 2 Mrd. Dollar per annum). Im Fiskaljahr 2005/06 waren es dann „schon“ 5,5 Mrd. Dollar (zum Vergleich: China 60 Mrd.). Dann erfolgte ein steiler Anstieg auf 50 Mrd. Dollar $(2009 / 10)$. Dieser enorme Anstieg geht überwiegend auf Investitionen und Spekulationen der Non Resident Indians (NRIs) aus den USA zurück und auf Überweisungen indischer Gastarbeiter aus den Golfstaaten. Ohne massive Kapitalinjektionen aus dem Ausland sind die ehrgeizigen Ziele der indischen Wirtschaft jedoch nicht zu verwirklichen.

Immer stärker investieren aber auch indische Firmen im Ausland. Besonders spektakulär gestaltete sich im Jahr 2006 die Akquisition des europäischen und bis dahin weltgrößten Stahlkonzerns Arcelor durch Mittal Steel. Noch größeres Aufsehen erregte die Übernahme von Corus (früher British Steel) durch Ratan TATA Ende Jänner 2007 um 9,3 Mrd. Euro. Was für ein Triumph der ,,boys“ über ihre früheren britischen „,master“! Markiert dies den Beginn des Überlebenskampfes von „Old Europe“ gegen die kommenden Weltmächte China und Indien? Experten wie jene der Boston Consulting Group bescheinigen indischen Managern exzellente Fähigkeiten und Leistungen wie hohe Problemlösungskapazität, Flexibilität und Innovationsfreude. Immer mehr kreative Forscher und Spezialisten aus Europa, auch aus dem deutschen Sprachraum, arbeiten bereits für indische Konzerne in Indien und anderswo (IinformationstechnologieBereich, Pharmaindustrie, Weltraumforschung etc.) 


\subsection{Entwicklungshemmnisse}

\subsubsection{Vernachlässigte Infrastruktur}

Als größte Schwachstelle der ökonomischen Entwicklung gilt die über Jahrzehnte sträflich vernachlässigte Infrastruktur. Dabei hatte Indien als Erbe der britischen Kolonialzeit ungleich bessere Ausgangsbedingungen als sein Rivale China, nämlich das größte Bahnnetz der Erde „under one management“ mit über $60.000 \mathrm{~km}$ und ein Straßennetz von 3,8 Mio. km. Zu den Problembereichen zählen der wegen fehlender Mittel immer wieder verschobene oder verzögerte Ausbau der Schienenwege und Straßen, die Modernisierung von Häfen und Flughäfen. Als besonders hemmend erweisen sich die immer stärker in Verzug geratene Stromerzeugung und Wasserversorgung, die Erneuerung und Ausweitung städtischer Infrastruktur etc. Inzwischen ist es Indien - wenn auch zu wenig und zu langsam - doch gelungen, ausländische Investoren für eine Reihe von Public-Private-Partnership-Projekten (PPP-Projekten) zu gewinnen.7)

\subsection{2 Ökologische Probleme}

Es existieren in Indien vielfältige ökologische Probleme. Die Umweltdegradation reicht von der Vernichtung der Lebensräume der Ureinwohner (Adivasi) durch Großprojekte nationaler und transnationaler Konsortien (Entwaldung, Schürfrechte) über massive Schwierigkeiten in der Landwirtschaft (Bodendegradation, Wasserverbrauch, globale Klimaveränderung) bis zu unmenschlichen hygienischen Lebensbedingungen der vielen Millionen von Slumbewohnern in den Megacities. Da tröstet nur wenig die Expertenmeinung, dass in China die Umweltproblematik noch viel schwerwiegender sei als in Indien.

Bei den Großkonferenzen zum Klimawandel (zuletzt in Kopenhagen [København]) fällt besonders unangenehm auf, dass ausgerechnet Indien sich vehement gegen eine Festschreibung seines künftigen Beitrags wehrt (Hansen 2010). Tenor seiner Gegenposition ist es, das Recht Indiens auf (nachholende) Entwicklung, auf größeres Wirtschaftswachstum zu schützen und zuerst die westlichen Industriestaaten in die (finanzielle) Verantwortung zu nehmen. Dabei ist Indien bereits nach China, den USA und Russland der viertgrößte Emittent von Treibhausgasen (Weltanteil 5\%), wenngleich der Pro-Kopf-Wert natürlich nur einen Bruchteil der hoch entwickelten Staaten ausmacht. Die Entwicklungsländer seien die Hauptopfer des Klimawandels, hätten ihn aber nicht verursacht. Dies war die Position der sogenannten BASIC-Staaten (Brasilien, Südafrika, Indien und China) in Kopenhagen.

Einige Nachbarstaaten Indiens, nämlich Nepal, Bangladesch und die Malediven, wollen Indien trotzdem in eine Führungsrolle im Kampf gegen den Klimawandel drängen. Dabei hat die indische Regierung die ökologische Problematik im Land so lange geleugnet, bis sich die katastrophalen Auswirkungen nicht mehr länger verheimlichen

\footnotetext{
7) Der Forschungsbericht „Infrastruktur Indien“ der Deutschen B AnK (2008) erläutert Defizite, Projekte und Investitionsprogramme der wirtschaftlichen und sozialen Infrastruktur und entwickelt ein Bewertungsmodell für mittelfristige Investitionen (mit möglichen PPP-Projekten) auf Bundesstaatenebene - http:// www.dbresearch.de
} 
ließen. Im Jahr 2007 wurde ein Klimarat gegründet, 2008 der Nationale Klimaaktionsplan veröffentlicht (GoI 2008). Selbst dieser Plan anerkennt den Vorrang des ökonomischen Wachstums vor Klimaschutz unter dem „Vorwand“ der Armutsbekämpfung.

Südasien gehört zu den Weltregionen mit der stärksten Gefährdung durch den Klimawandel. Beispielhaft seien nur das Abschmelzen der Gletscher im Himalaja, die Ganges, Brahmaputra und Indus speisen, oder Ausfälle im Agrarsektor durch unregelmäßigere Monsunzyklen genannt. ZingeL (2009) analysiert den engen Konnex von Umweltproblemen mit wirtschaftlichen, sozialen und religiösen Konflikten.

\subsubsection{Achillesferse Landwirtschaft}

Auch im 21. Jh. bleibt klar, dass Indien noch immer ein Agrarland ist. Über $60 \%$ der Erwerbstätigen arbeiten weiterhin im Agrarsektor. Ein Großteil von ihnen sind Kleinbauern und Tagelöhner, die sich mit einem Hungerlohn durchfretten. Viele enden in Schuldknechtschaft. Dienstleistungssektor (20-25\%) und Industrie (10\%) sind nicht in der Lage, die Abwanderungswilligen zu absorbieren. Der Anteil der Landwirtschaft am BIP hat aber ständig abgenommen und liegt bei derzeit 17,5\% (Dienstleistungen $53 \%$, Industrie 28\%). Indien hat seit 1991 einschneidende Kürzungen der geplanten Investitionen für ländliche Bereiche vorgenommen. Im Jahr 1991 entsprachen die staatlichen Ausgaben noch 14,5\% des BIP, 2005 nur noch 5,9\%. Dies ist mitverantwortlich für die schlimmste Agrarkrise seit dem Ende der Grünen Revolution.

Traditionell leidet die Landwirtschaft an struktureller Unterentwicklung, mangelnder Produktivität und einem Überbesatz mit Arbeitskräften. Von 1997 bis 2007 hat sich die Verschuldung der indischen Bauern fast verdoppelt, was zehntausende Selbstmorde zur Folge hatte. Die agrarpolitische Deregulierung ließ die Preise für Inputs wie Saatgut und Düngemittel stetig ansteigen. Millionen Bauern wurden im Sinn eines exportorientierten Anbaumodells zum Anbau von „cash crops“ (etwa Baumwolle) animiert, und nach der Umstellung verfielen die Weltmarktpreise. Die Kommerzialisierung und Privatisierung der Landwirtschaft führte insgesamt zu einem enormen Anstieg der Lebenshaltungskosten. Für die Masse der Bevölkerung blieben die Einkommen „indisch“, die Preise wurden globalisiert. Indiens größte Verlierer sind bisher die im ländlichen Raum lebenden Menschen, insgesamt rund 800 Millionen.

\subsubsection{Weitere Problembereiche}

Weitere negative Faktoren sind mit dem hohen Haushaltsdefizit und mit der Auslandsverschuldung gegeben. Relativiert werden diese Probleme jedoch durch einen steil ansteigenden Exportüberschuss bei Dienstleistungen und umfangreiche Rücküberweisungen aus den Golfstaaten und den USA. Mit 52 Mrd. Dollar „remittances“ (Weltbank 2009) steht Indien hier weltweit an der Spitze vor China und Mexiko. Gleichzeitig hat Indien in den letzten Jahren auch aus der großen Krise des Jahres 1991 gelernt und bedeutende Devisenreserven angehäuft (2006 bereits 155 Mrd. Dollar).

Größere Hemmnisse stellen nach wie vor auch die berüchtigte indische Bürokratie sowie die Korruption in allen Bereichen von Politik, Verwaltung und Wirtschaft dar. Auf dem Korruptionsindex von TRANSPARENCY INTERNATIONAL findet sich Indien im Jahr 
2009 unter 180 erfassten Ländern auf Platz 84. Es bleibt der subjektiven Bewertung überlassen, ob damit das Glas halbvoll oder halbleer ist.

\subsection{Entwicklungschancen}

\subsubsection{Das untypische Entwicklungsmodell - der rasante Aufstieg von Informationstechnologie (IT) und Business Process Outsourcing (BPO)}

MüLler \& Rauch (2008, S. 3) bringen es auf den Punkt: „Den Lehrbüchern entsprechend entwickeln sich periphere Volkswirtschaften durch die Kapitalisierung der Landwirtschaft über die Massenproduktion von Konsumgütern, deren Produktion gering qualifizierte Arbeitskraft benötigt, zu einer soliden Industriewirtschaft, bevor der Dienstleistungssektor die Führung des wirtschaftlichen Wachstums übernimmt. Diese Lehre hat Indien auf den Kopf gestellt.“ Wie in einem Zeitraffer überspringt ein riesiges, aber armes Land der Dritten Welt einige Stufen der Entwicklung und wird innerhalb kürzester Zeit zum gefürchteten „global player“ in der Hochtechnologie. Die Zwischenstufen massiver Industrialisierung werden damit sozusagen „ausgelassen“.

Im dritten Jahrtausend ist Wissen die bedeutendste Ressource für Erfolge in der globalisierten Wirtschaft. Die Exporte wissensbasierter Industrien haben die wichtigsten Ausfuhrprodukte früherer Jahrhunderte - Tee, Gewürze und Textilien - längst übertroffen. Schrittweise errang schon seit den 1970er Jahren indische Software immer größere Problemlösungskompetenzen und damit Anteile am Weltmarkt. Die Mehrzahl der 500 führenden Unternehmen der USA (nach dem Fortune-Index) hat ihre Software-Agenden ab dem Jahr 2000 nach Indien verlagert.

Entstand erst 1990 der erste Software Technology Park in Bengaluru, so ist Indien heute die führende Nation in der Softwareproduktion und -anwendung. Durch die 1990er Jahre wuchs diese Sparte in Sprüngen von $50 \%$ pro Jahr. Jeder dritte IT-Fachmann weltweit ist heute indischer Herkunft (allein im kalifornischen Silicon Valley vier von zehn Experten). Zu den Weltfirmen zählen Infosys, Wipro und Tata Consultancy Services (TCS). Es boomen insbesondere die Dienste der „,back offices“ und der „call centers “. Diese übernehmen sehr vielfältige Aufgaben wie Buchführungen, Steuerbescheide, Kundenbetreuung, Forderungsabwicklung, Werbung usw. Der riesige Pool an kompetenten Fachkräften mit fließender Beherrschung des Englischen hat entscheidend zu diesem Boom beigetragen.

Neben der Software-Entwicklung und -Produktion ist Indiens zweiter Entwicklungsmotor die Übernahme kompletter unterstützender Geschäftsprozesse, das „Business Process Outsourcing“ (BPO) (Genaueres dazu in DB Research 2005). Die Anbieter von BPO und IT-Dienstleistungen erweitern ständig ihre Angebotspalette und erklimmen immer höhere Stufen der Wertschöpfungsleiter. Immer bedeutender werden wissensbasierte Dienstleistungen („Knowledge Process Outsourcing“, KPO) von der Risiko-Modellierung für Finanzdienstleister bis zur Remote-Beratung durch Fachärzte.

Die National Association of Software and Services Companies (NASSCOM) liefert dazu die neuesten Aussagen: „Over the past decade, the IT-BPO sector has become ... the global brand ambassador for India“ (2010, S. 5). Im Finanzjahr 2009/10 hat 
dieser Sektor 73 Mrd. Dollar an Wertschöpfung erbracht und beschäftigte 2,3 Mio. Menschen direkt und 8,2 Mio. sekundär durch das Erbringen von Zusatzleistungen. Sein Anteil am BIP ist von 1,2\% im Jahr 1998 auf nun 6,1\% gewachsen, sein Anteil an allen Exporten von $4 \%$ auf $26 \%$ mit einem Wert von über 50 Mrd. Dollar gestiegen. $61 \%$ des Exportwerts gingen in die USA. Trotz zunehmender Konkurrenz soll die Wertschöpfung bis zum Jahr 2020 auf 225 Mrd. Dollar gesteigert werden.

\subsubsection{Spitzenleistungen in Forschung und Technologie}

Das indische Wirtschaftswachstum beruht aber längst nicht mehr nur auf dem rasanten Wachstum der IT-Dienste. In die Weltspitze stößt Indien in verschiedenen Bereichen vor, etwa auch in der Biotechnologie, in der Pharmaindustrie (Letztere ist bereits die viertgrößte der Erde, die über $40 \%$ aller Generika produziert.), in Nanotechnologie und Raumfahrt.

Greifen wir ein weiteres Prunkstück der indischen Wissenschafts- und Technologieleistungen heraus, die Raumfahrt. Sie wurde mit ersten Versuchen im Jahr 1963 gestartet, 1969 erfolgte die Gründung der Indian Space Research Organisation (ISRO). Im Jahr 1975 startete der erste indische Satellit mit sowjetischer Trägertechnologie, 1980 der erste mit indischer Trägerrakete. Im Jahr 1984 verbrachte Rakesh SHARMA als indischer Kosmonaut acht Tage an Bord der sowjetischen Raumstation Saljut 7.

Indien entwickelt seither routinemäßig eigene Trägerraketen und Satelliten mit immer höherer Leistung, deren Gestehungskosten weit unter jenen der National Aeronautics and Space Administration (NASA) oder der European Space Agency (ESA) liegen (etwa Indian National Satellite System - INSAT; Indian Remote Sensing Satellite System - IRS; Geosynchronous Satellite Launch Vehicle - GSLV etc.). Derzeit arbeiten etwa 20.000 Mitarbeiter in einer Reihe von Forschungsstationen landesweit. Die größte Einrichtung ist das Vikram Sarabhai Space Center (VSSC), benannt nach dem Vater der indischen Raumforschung, mit 6.000 Spezialisten in Triwandrum [Tiruvanantapuram] im Bundesstaat Kerala. International bekannt ist inzwischen auch das Raketenstartgelände von Sriharikota in Andhra Pradesh, von wo am 22. Oktober 2008 Indiens erste Raumsonde Chandrayaan I (das Sanskrit-Wort chandra bedeutet ,Mondgott') erfolgreich in die Mondumlaufbahn startete. Indien ist fest entschlossen, zwischen 2016 und 2020 zwei Gaganauten (das Sanskrit-Wort gagan bedeutet ,Himmel') in den Weltraum zu senden. Weniger spektakulär, aber nützlich, sind dutzende installierte Erdbeobachtungssatelliten (Wetterbeobachtung, Landwirtschaft, Katastrophenschutz, Bildungsprogramme etc.). Natürlich existiert neben der zivilen auch eine militärische Komponente mit Aufklärungs- und Navigationssatelliten.

Im Gefolge dieser Hochtechnologiebranchen erfahren jetzt auch Massenproduktionen von Konsum- und Investitionsgütern einen Aufschwung: im Automobilbau - zunächst noch mit Joint Ventures großer Hersteller, aber zunehmend autark in Eigenentwicklungen (Tata-Nano) - im Anlagenbau, in der Textilbranche. Die größte Filmindustrie der Welt („Bollywood“) produziert 900 Filme im Jahr, hat 2009 bereits 10 Mrd. Dollar umgesetzt und wurde inzwischen international zum Exportschlager. Glänzende Aussichten hat der gerade einsetzende Medizintourismus (Spitzenleistungen zu einem Bruchteil der Kosten in Europa oder den USA). 


\section{Stabile Demokratie - fragile Sozialstruktur}

\subsection{Indiens Demokratieentwicklung}

Indien, die „funktionierende Anarchie“ (nach einem Ausspruch des legendären amerikanischen Botschafters John K. GALBRAITH) stützt sich auf eine robuste und in ihrer Art einzigartige Demokratie. Die „,größte Demokratie der Welt“ erfüllt die Inder mit Stolz und gehört zu den Trumpfkarten des Landes im Pokerspiel der aufsteigenden Mächte.

Die Voraussetzungen der Demokratieentwicklung waren günstig. Der Rückzug des britischen Empire erfolgte schrittweise im Verlauf von 50 Jahren und ermöglichte damit einen langen Reifeprozess der Unabhängigkeitsbewegung, ihrer Führer und politischen Diskurse. Die Kader des kolonialen Apparats mutierten zum Rückgrat der neuen Indischen Union. Beamte, Armee und Polizei übernahmen die Spielregeln der „Westminster-Demokratie“ ohne politische Ambitionen. Aus der führenden Kraft des Unabhängigkeitskampfes, der Kongress-Bewegung, wurde die Kongresspartei durch vier Jahrzehnte die „staatstragende und staatsgetragene Partei“ (ROTHERMUND). Ihre Politik der Mitte basierte auf den drei Eckpfeilern Demokratie, Säkularismus und Sozialismus (Anti-Kolonialismus). Politologen bezeichnen diese Rolle als „one dominant party system“, d.h. ein Mehrparteiensystem unter Vorherrschaft des „Kongress“ in allen Bereichen des öffentlichen Lebens. Die Ausschaltung des Parlaments durch Indira GANDHI in den Jahren 1975-77 ist das schwärzeste Kapitel der indischen Zeitgeschichte, aber von der jungen Generation bereits vergessen .

In den 1980er und 1990er Jahren erfuhr dann der Hindu-Nationalismus („Hindutva“) einen starken Aufschwung, der dem „Kongress“ unter der Leitung der Bharatiya Janata Party (BJP, „Indische Volkspartei““) die politische Führungsrolle von 1998 bis 2004 entriss. Die dritte ideologische Säule, die heute immer wichtiger wird, ist die „erfolgreiche Selbstermächtigung der Unterprivilegierten“ (MüLLER 2007, S. 51), von Dalits (Unberührbaren), Adivasi (Stammesbevölkerung), religiösen und regionalen Minderheiten. Es ist faszinierend zu beobachten, wie Abermillionen Analphabeten von ihrem Wahlrecht Gebrauch machen. Verbesserte Schulbildung, Medien etc. führten schrittweise zur Mobilisierung der „underdogs“. Als Folge davon entstand, vor allem seit 1990, eine Fülle sekundärer Parteien auf lokaler, regionaler oder kastendominierter Basis.

\subsection{Die Wahl 2009 und ihre Vorbildwirkung}

Auch nach mehr als 60 Jahren ist das Ende der demokratischen Institutionen und der Staatsverfall, wie von nicht wenigen Auguren angekündigt, nicht eingetroffen. Im Gegenteil - die Fähigkeit der politischen Eliten, auf unterschiedliche Strömungen zu reagieren, und die Ausweitung der politischen Partizipation auf immer breitere Bevölkerungsgruppen haben die Legitimität der indischen Demokratie gestärkt. Repräsentative Umfragen zeigen, dass nahezu 70\% der Befragten heute die politischen Institutionen (nicht die Politiker!) positiv beurteilen - gegenüber 45\% im Jahr 1971. 
Bis zu zwei Drittel der armen Wähler sind davon überzeugt, dass ihre Stimme zählt. Nur $11 \%$ meinten, ein starker Führer wäre besser als Parteien, Wahlen und Parlamentarismus an sich. Im Lauf der Jahre gelang es, selbst ursprünglich gewaltbereite Gruppen in das politische System zu integrieren.

Bei den im April/Mai 2009 abgehaltenen Wahlen zur 15. Legislaturperiode (2009_ 2014) in das Unterhaus (Lok Sabha) wurde dies eindrucksvoll bestätigt (Nissel 2009). Von 714 Mio. Wahlberechtigten (!) gaben 428 Mio. (60\%) ihre Stimme ab. 369 Parteien stellten 8.070 Kandidaten auf. Im neuen Parlament sind 37 Parteien und neun unabhängige Abgeordnete vertreten. Gegen alle Prognosen hat die Nation für Kontinuität und Stabilität der bisherigen Regierungskoalition von „Kongress“ und verbündeten Parteien votiert. Die Oppositionsparteien von Mitte-Rechts (BJP, Jana SANGH) bis weit Links (sämtliche Linksparteien) erlitten schwere Verluste. Im Vergleich zu seinen Nachbarn - den „failing states“ von Afghanistan bis Myanmar - und den jüngsten traumatischen Entwicklungen in Pakistan und Sri Lanka wirkt Indiens innenpolitischer Zustand wie ein Leuchtturm auf viele Staaten der Dritten Welt. Die Botschaft lautet: Demokratie ist kein Luxusgut des Westens, vielmehr bringt die Aktivierung von demokratischen Werten und Normen langfristig Fortschritt, Frieden und Freiheit auch für Entwicklungs- und Schwellenländer. Im Wettlauf der beiden asiatischen Giganten könnte dies eines Tages das Pendel zu Gunsten Indiens ausschlagen lassen.

\subsection{Problematische Seiten der Demokratie}

Der Staat versucht seit Jahrzehnten, die gewaltigen Ungleichheiten der Gesellschaft durch Mittel der ,positiven Diskriminierung“ auszugleichen. Die „Reservierung“ von Plätzen für die am stärksten Benachteiligten im Staatsdienst, an den Universitäten usw. macht viel böses Blut. Festgelegt sind 15,5\% aller Stellen für Scheduled Castes (SCs), also für eigentlich früher so apostrophierte „outcasts“ oder Unberührbare, heute Dalits genannt, und 8\% für Scheduled Tribes (STs), ,gelistete Stämme“, Ureinwohner oder Adivasi.

Das Konzept stammt noch aus der Kolonialzeit. Diese im Ranking ganz unten stehenden Gruppen wurden sozial und räumlich über alle Generationen stigmatisiert und ausgegrenzt. Inzwischen haben SCs eigene Parteien gebildet und kämpfen STs um territoriale Autonomie (erfolgreich im Jahr 2000 mit Bildung der neuen Bundesstaaten Chattisgarh und Jharkhand).

Weniger drastisch, trotzdem problematisch gestaltete sich die Diskriminierung der Other Backward Classes (OBCs). Seit den Empfehlungen der sogenannten MandalKommission zu Anfang der 1990er Jahre wurden über 3.700 solcher Gruppierungen Vergünstigungen eingeräumt. Diese zunächst nur statistische Kategorie wurde damit zur sozialen Gruppe der OBC, die in manchen Bundesstaaten schon über die Hälfte der Bevölkerung umfasst.

Damit gewann etwa eine neue Partei der Unberührbaren, die Bahujan Samaj Party (BSP), im Jahr 2007 die Regionalwahlen in Uttar Pradesh, dem Bundesstaat mit 170 Mio. Einwohnern. Die früher erfolgreiche „BMW“-Strategie der Kongresspartei (BMW = Brahmins, Muslims and Weaker Sections) ist hier nicht mehr aufgegangen, 
sondern von der BSP erfolgreich kopiert worden. Die politische Auseinandersetzung gerinnt immer öfter zum bloßen Lippenbekenntnis. Vielmehr geht es um die Zuteilung von Privilegien, Quoten und Subventionen an unterschiedliche Interessengruppen. Trotz starker Zweifel, ob diese Zuwendungen die wirklich Bedürftigen erreichen, werden dadurch immer neue Begehrlichkeiten geweckt. Erfolgreiche Politiker mobilisieren die entsprechenden Wählergruppen - Weltanschauungen, Wahlprogramme sind unwesentlich. Verbreitet ist auch der dynastische Faktor, bestens verkörpert in der NeHRU-GANDHI-Tradition über mittlerweile vier Generationen. Klientelismus und Korruption gehören zum politischen Alltag und durchziehen ebenso sämtliche Verwaltungs- und Wirtschaftsbereiche.

\subsection{Reibungen zwischen Kastenstruktur und sozialem Status}

Es ist im Rahmen dieser Arbeit nicht möglich, auf die extrem vielschichtige Problematik der indischen Religions- und Sozialstruktur einzugehen. Es mögen daher einige Andeutungen genügen, inwieweit diese Faktoren in die Politik hineinwirken.

Die Kastenordnung ist das Jahrtausende überdauernde traditionelle soziale Gefüge Indiens - ursprünglich eine strenge Hierarchie, in der der Platz des Einzelnen durch die Geburt unverrückbar bestimmt ist, mit strikten Essensregeln, Berufszuweisungen und Heiratsgeboten. Obwohl Diskriminierung aufgrund der Kastenzugehörigkeit genauso verfassungswidrig ist wie hinsichtlich Konfession, Rasse oder Geschlecht, bestimmt das Kastendenken das Alltagsleben der Inder weiterhin zutiefst.

In der rituellen Hierarchie stehen die Brahmanen (Priesterkaste) an der Spitze, gefolgt von Kriegern, Kaufleuten, Bauern und den „Unberührbaren“ außerhalb der Ordnung. Die Unterdrückung Letzterer kann strafrechtlich verfolgt werden, ist aber im Alltag jederzeit präsent. Das Bekenntnis zur eigenen Kaste und das Endogamiegebot (Heirat nur innerhalb der Kaste) wird von einer überwältigenden Mehrheit aller Hindus gelebt.

Unter den Briten wurden die schreibkundigen Brahmanen zur Stütze der Kolonialverwaltung. Auch in der Indischen Union sind sie noch immer im Machtgefüge weit überrepräsentiert. Mit 3,5\% Anteil an Bevölkerung besetzen sie die Hälfte der Parlamentssitze, 78\% der Posten im Justizwesen und stellen 80 bis $90 \%$ der Universitätsprofessoren. Die Basis ihrer Macht, ihrer Privilegien und ihres Status erscheint aber heute als gefährdet. Es ist daher zu verstehen, dass sie den politischen Aufstieg der vordem Unterprivilegierten mit aller Macht bekämpfen.

Sozialer Status war im System immer zugeschrieben und nicht durch Leistung erworben. Moderne und Postmoderne erschüttern zunehmend dieses Gefüge durch erzwungenes Zusammenleben (z.B. innerhalb der Megastädte), neue Berufsfelder, Lohnabhängigkeit usw. Doch Kasten verschwinden keineswegs. Im Gegenteil, sie werden vielleicht individuell unterhöhlt, Kasten niedrigen Rangs mit vielen Wählern werden jedoch zu begehrten „,dominant castes“. Die früher Benachteiligten ziehen jetzt Vorteile aus dem System. Kastenstruktur wandelt sich von einer streng vertikalen Hierarchie immer mehr zu einer horizontal verschachtelten Ausformung von Interessengruppen. 


\section{Außenpolitik, Atommacht und geopolitische Ambitionen}

\subsection{Neues Selbstbewusstsein}

Mit dem ökonomischen Aufstieg und der verstärkten internationalen Anerkennung erfolgt auch ein Perspektivenwechsel in der indischen Außenpolitik mit Blick auf die mögliche Rolle Indiens in einer multipolar geprägten Welt. Im Gegensatz zu anderen Mächten haben Indiens Politiker und Vordenker bisher keine spezifische außenpolitische Doktrin entwickelt und schon gar kein „grand design“ wie die USA. Es wurden eher pragmatisch über die Jahre viele kleinere Schritte gesetzt, um das politische Gewicht des Landes auf der Weltbühne zu vergrößern (ausführlicher dazu bei Nissel 2008). Gleichwohl zeichnen sich heute Kernelemente eines neuen Politikverständnisses wie auch wachsende Selbstsicherheit im internationalen Umgang mit der Staatengemeinschaft ab. Dazu zählt nach Jahrzehnten gegenseitigen Misstrauens, ja geradezu feindseligen Gegeneinanders, ein ständig intensiviertes Verhältnis zu den USA, sowie - und dies ist noch erstaunlicher - eine deutliche Korrektur und Verbesserung der Beziehungen zur Volksrepublik China. Obwohl Russland nach wie vor wichtigster Partner bei Waffeneinkäufen ist, scheint die lange unverbrüchliche Freundschaft unter den geänderten Bedingungen etwas zu verblassen.

Deutlich größere Probleme ergeben sich für Indien aus seiner „,natürlichen“ Führungsrolle als Regionalmacht Südasiens. Die schwierigste und weiterhin virulente Frage ist nach wie vor diejenige der Beziehungen zu Pakistan. Drei Kriege, ein gerade noch verhinderter Atomkrieg (1999), die ungelöste Kaschmirfrage, der Anschlag pakistanischer Terroristen im November 2008 in Bombay - die Liste ist lang. Sekundär, trotzdem unangenehm, sind die jahrelangen Querelen mit Bangladesch, die blutigen Bürgerkriege in Sri Lanka, Nepal und Afghanistan. Die von Indien gewünschte Rolle des „freundlichen Hegemons“ in Südasien wird von den Nachbarstaaten sehr schnell als Bevormundung und Einmischung in die inneren Angelegenheiten gesehen.

\subsection{Neue Geopolitik}

Dafür blickt Indien weit über den südasiatischen Tellerrand hinaus und bemüht sich verstärkt um Kontakte mit Südostasien und Kernstaaten der Entwicklungsländer in Afrika und Lateinamerika. Ähnlich wie und in Konkurrenz zur VR China versucht Indien einerseits gegen seinen großen asiatischen Rivalen zu punkten und gleichzeitig notwendige Ressourcen für den Aufstieg zur Weltmacht zu sichern. Allerdings weist China auch in dieser Hinsicht bereits (oder noch) einen bedeutenden zeitlichen und materiellen Vorsprung auf. Sehr interessant ist die neue Doktrin, im Indischen Ozean eine weltpolitische Schutz- und Kontrollfunktion übernehmen zu wollen. Dieses marenostro-Konzept soll von der Straße von Hormus bis Malakka reichen, die Erdöltransporte zwischen dem Persischen oder Arabischen Golf, Afrika, Indien und Japan sichern und natürlich ebenso den Expansionsdrang Chinas eindämmen. Der mächtige Ausbau von Marine und Luftwaffe ist langfristig auf dieses Oberziel ausgerichtet. 
In der indischen Geostrategie lassen sich somit drei Interessenssphären ausmachen. Der innere Kreis besteht aus den Nachbarstaaten am Subkontinent, für die Indien immer eine Führungsrolle beansprucht hat - aufgrund seiner Größe, seiner ökonomischen Bedeutung und der „gelebten Demokratie“ sicher nicht ohne Berechtigung, stützt sie sich doch auf drei Viertel der Bevölkerung und des BIP und zwei Drittel des Außenhandels in Südasien. Priorität wurde dabei seit Jahrzehnten den Bemühungen eingeräumt, die Einflussnahme anderer Mächte zu minimieren. Zum Beispiel wird Kaschmir als bilaterales Problem behandelt, während Pakistan sich durch Internationalisierung eine Konfliktlösung erhofft. Allerdings schreckte Indien wiederholt nicht vor politischem Druck und militärischem Eingreifen zurück: 1971 durch aktive „Mithilfe“ bei der Spaltung Pakistans und der Staatsgründung von Bangladesch, 1988 bei der Niederschlagung eines Staatsstreichs auf den Malediven, 1987-1990 erfolglos trotz starker Präsenz in Sri Lanka.

Der zweite Ring umfasst das weitere Umfeld in Asien und im Bereich des Indischen Ozeans, wo Indien zuvorderst daran gelegen ist, eine Machtbalance in einem zukünftigen multipolaren weltpolitischen System zu entwickeln und aufrechtzuerhalten. In erster Linie geht es darum, der befürchteten Hegemonie der VR China einen Riegel vorzuschieben.

Und drittens geht es um den gesamten Erdball (und Weltraum?), auf dem Indien seinen „Platz an der Sonne“ unter den Großmächten als ,global player“" und Mitgestalter der internationalen Friedens- und Sicherheitsbemühungen einzunehmen gedenkt. Deshalb fordert Indien auch seit geraumer Zeit einen ständigen Sitz im Sicherheitsrat der Vereinten Nationen (was bisher am Veto Chinas scheiterte).

Noch während der Phase des Kalten Krieges wurde Indien aufgrund des eigenen Politikverständnisses und seiner ökonomischen Nabelschau nicht einmal als zweitklassige Macht eingestuft. Mit dem Zerbrechen der Sowjetunion stand das Land plötzlich auf der Seite der Verlierer. Die „größte Demokratie der Welt“ hatte ironischerweise das Freundschaftsbündnis mit der kommunistischen Supermacht praktiziert, die USA mit Pakistan, einem Land, das mehrfach zwischen Unregierbarkeit im Chaos und Militärdiktatur wechselte.

Durch die veränderten innen- wie außenpolitischen und ökonomischen Rahmenbedingungen wurden sehr rasch neue Zielsetzungen möglich, die vorher undenkbar waren - vor allem der Positionswechsel hin zum friedlichen Wettstreit mit den ehemaligen deklarierten Gegnern USA und China. Schlagartig veränderte „9/11“ die Weltbühne im gemeinsamen Kampf gegen den Terrorismus. Die geopolitischen Leitbilder eines einfachen Freund-Feind-Schemas haben heute ausgedient. Die indische Außenpolitik konnte sich damit neu formieren und in eine Reihe bi- und multilateraler Vereinbarungen eintreten. Es zeichnet sich gleichzeitig eine neue Bewertung Indiens in der Welt und der Welt durch Indien ab. Noch ist nicht entschieden, wie China und Indien in Zukunft miteinander umgehen, ob die traditionelle Rivalität Oberhand gewinnt oder sich sogar neue, bis vor kurzem undenkbare Formen der Kooperation zwischen den beiden Riesen, etwa auf Kosten Japans oder Koreas, herausbilden (wie das vielleicht an der rasanten Zunahme des bilateralen Handels ablesbar ist, der noch $201050 \mathrm{Mrd}$. Dollar erreichen soll). 


\subsection{Atommacht}

Die Rolle Indiens in der Welt wird nicht zuletzt durch seinen Status als Atommacht mitbestimmt. Der Beginn des Nuklearwaffenprogramms kann als Folge der schmerzlichen Niederlage im indisch-chinesischen Grenzkrieg des Jahres 1962 und der ersten chinesischen Nukleartests im Jahr 1964 gesehen werden. Indiens erster „friedlicher“ Atomtest fand im Mai 1974 statt. Gegner und Befürworter einer atomaren Bewaffnung standen sich in der indischen Politik jahrzehntelang gegenüber. Bis 1998 leugnete Indien offiziell den Besitz von Atomwaffen und sprach immer nur von einer „nuklearen Option“, die es sich offen halten wollte. Bis heute hat Indien nicht das Abkommen über die Nicht-Weiterverbreitung, die Non Proliferation Treaty (NPT) unterzeichnet, die es als diskriminierend ablehnt (an der Seite von Pakistan, Israel und Nordkorea!).

Unter der Regierung der hindunationalistischen, rechtsgerichteten BJP-Partei wurde (plötzlich und unerwartet) am 11. Mai 1998 eine Serie von sechs Nukleartests gestartet, die gleich als „hinduistische“ Bomben hochgejubelt wurden. Nur wenige Tage später antwortete Pakistan mit einer Serie von ebenfalls sechs ,islamischen“ Bomben. Die strenge Symmetrie beweist die hochgradige Projektion der gegenseitigen Ängste. Es ist wiederum eine Ironie, dass der Vater der friedlichen Atomenergienutzung in Indien ein Parse war, Homi BABHA, und das „mastermind“ der Raketentechnologie, der spätere Staatspräsident Abdul KALAM, ein Moslem (Indien ist seit der Gründungsstunde ein säkularer Staat!). Die daraufhin verhängten internationalen Sanktionen wurden nach drei Jahren wieder aufgehoben und waren nicht sehr schmerzvoll. Die Strategie nuklearer Abschreckung bestimmt seither das Handeln Indiens und Pakistans.

Ununterbrochen wird an immer weiteren Serien von Raketen aller Reichweiten gebastelt, für alle Waffengattungen. Delhi bezeichnet dies im Rahmen der indischen Nukleardoktrin (1999) als in Übereinstimmung mit dem Prinzip einer glaubwürdigen Minimalabschreckung („minimum credible deterrence“) und des Nichtersteinsatzes von Nuklearwaffen. Zahlenangaben zu einsatzfähigen Nuklearsprengköpfen schwanken zwischen 50 und 150 (Pakistan 30-60).

Umstritten ist unter Experten bis heute die Frage, ob die atomare Bewaffnung der „verfeindeten Brüder“ diese an weiteren Waffengängen gehindert oder das Aggressionspotenzial noch gesteigert hat (exzellent dazu KAPUR \& GANGULY 2010). Zu einem Triumph der indischen Diplomatie wurde dann die endgültige Freisprechung durch die USA anlässlich des Staatsbesuchs von Präsident Bush jr. im März 2006, bei dem Indien die friedliche Nutzung der Kernenergie erlaubt und es zugleich als (militärische) Atommacht anerkannt wurde. Dies erscheint paradox, denn Indien tauchte damit aus der nuklearen Quarantäne auf und wird nun wie ein Mitglied des Atomwaffensperrvertrags behandelt - entgegen allen bisherigen Abmachungen und trotz langjähriger Versuche zur Eindämmung der Verbreitung von Atomwaffen. Das entspricht einer bisher einzigartigen Ausnahmeregelung. Man kann sie geopolitisch damit erklären, dass die USA die strategische Partnerschaft mit Indien als Gegengewicht zum aufkommenden „wirklichen“ Rivalen um die Weltherrschaft, China, aufbauen wollen. 


\subsection{Aufrüstung und Entwicklungszusammenarbeit}

Mahatma GANDHIs Vermächtnis, die indische Politik von Ahimsa, dem Prinzip der Gewaltlosigkeit, leiten zu lassen, scheint heute Lichtjahre von der Realität entfernt. Das „neue“ Indien setzt nicht nur auf atomare Abschreckung, sondern gleichermaßen auf massiven Ausbau der konventionellen Militärmaschinerie. Weltmacht kann nur ein Staat werden, der politisch, ökonomisch - und eben auch militärisch - Signale der Stärke und Unbezwingbarkeit aussendet. ${ }^{8)}$

Indien kann gegenwärtig noch nicht mit der massiven Aufrüstung der chinesischen Streitkräfte mithalten, ist aber trotzdem zweitgrößte Macht Asiens, größter Waffenimporteur nach China (kontinuierlich seit 2004) und viertgrößte Militärmacht der Erde. Die Armee hat 1,1 Mio. Soldaten unter Waffen, die Luftwaffe 170.000 und die Marine 55.000. Dazu kommen paramilitärische Kräfte in gleicher Größenordnung. In den letzten Jahren stiegen die Militärausgaben überproportional jeweils zwischen 13 und 32\% und betragen 2009/2010 bereits 30 Mrd. Dollar (2,35\% des BIP, China im Vergleich 70 Mrd.). Detaillierte Angaben liefern die renommierten Institute IISS (International Institute for Strategic Studies) und SIPRI (Stockholm International Peace Research Institute). Noch immer ist Russland wichtigster Waffenlieferant mit $70 \%$ Anteil (Prunkstück ist der gemeinsam konzipierte Überschallflugkörper Brahmos, benannt nach den Flüssen Brahmaputra und Moskva.), für Hochtechnologie jedoch Israel (Raketenfrüherkennungs- und Abwehrsysteme).

Indien produziert aber bereits $35 \%$ seiner Rüstungsgüter selbst, angestrebt werden $70 \%$. Die Marine liegt an fünfter Stelle weltweit und wird besonders gefördert. Sie soll bis 2022160 Schiffe umfassen, darunter drei von Flugzeugträgern angeführte Flottenverbände, unter anderem mit Atom-U-Booten. Die Luftwaffe umfasst 850 Kampfjets unterschiedlicher Bauart. Um einen Jahrhundertauftrag über 126 Maschinen der neuesten Generation im Wert von 12 Mrd. Dollar bewerben sich alle großen Produzenten der Erde.

Das hohe Ansehen der Militärs hat diese jedoch nie verleitet, aktiv in die indische Innen- oder Außenpolitik einzugreifen, auch dies in deutlichem Gegensatz zu den Nachbarn oder anderen ,emerging powers“, etwa den BRIC-Partnern.

Mittel- bis langfristig wird Indien damit nicht nur die strategischen Planspiele der großen Mächte zunehmend beeinflussen, sondern sich auch selbst in die geopolitischen Entwicklungen zumindest in Asien und im Bereich des Indischen Ozeans verstärkt einschalten.

Geopolitik betreibt Indien auch noch auf einer anderen, zivilen Ebene. Es bringt sich immer stärker als ,emerging donor“ in die internationale Entwicklungszusammenarbeit ein, wandelt sich zunehmend vom Armenhaus zum Geberstaat als wichtiger Vertreter der neuen Süd-Süd-Kooperation. Die Ziele indischer Entwicklungszusammenarbeit leiten sich direkt aus den außen- und wirtschaftspolitischen Interessen ab und ordnen

\footnotetext{
8) Der Autor dankt Herrn ObstltdG Mag. Siтzwohl für die Bereitstellung der Unterlagen „Militärstrategische Beispiele - Indien“ im Rahmen des 18. Generalstabslehrganges des ÖBH im Februar 2010. Die aufwändig gestaltete Homepage der indischen Armee ist abrufbar unter http://indianarmy.nic.in/Index. aspx? flag $=$ lfc ULYFibe $Q$
} 
sich bisher nicht den Vorgaben und Verfahrensweisen der OECD-Mitglieder unter. Indien tritt deshalb verstärkt in rohstoffreichen Staaten Zentralasiens und Afrikas auf und offeriert einen Mix an attraktiven wirtschaftspolitischen ,incentives“ (Investitionen, Handel, Technologietransfer) gekoppelt mit politischer Unterstützung auf dem internationalen Parkett.

\section{Das ,andere“ Indien - Armut, Hunger, Widerstand}

\subsection{Alltag der Globalisierungsverlierer - Armut und Hunger}

Bisher war überwiegend von den vielfältigen positiven Veränderungen eines „shining India“" (ehem. Wahlslogan der BJP) die Rede. Doch wäre es völlig verfehlt, nicht auch die Kehrseite der Medaille anzusprechen. Vom ökonomischen Aufschwung hat bisher nur eine Minderheit profitiert, nämlich die Eliten und Mittelschichten. Die große Mehrheit der indischen Bevölkerung (etwa drei Viertel) sind Globalisierungsverlierer, für die Hunger und Armut noch immer bittere Alltagswirklichkeit bedeuten. Die energische Bekämpfung des Massenelends bleibt die größte Herausforderung indischer Politik überhaupt. Das Problembewusstsein dafür wird stärker. Doch ist der Wille zur politischen Umsetzung (unter neoliberalen Prämissen) wirklich stark genug? Eines erscheint als ganz klar: ohne Lösung der Armutsproblematik wird Indien niemals den Status einer Weltmacht beanspruchen können.

Das schiere Ausmaß von Armut und Hunger ist schockierend, die einzelnen Dimensionen der Verelendung sind es noch mehr. ${ }^{9)}$ Eine führende Kritikerin, Utsa PATnAIK, stellt die These auf, dass die neoliberale Politik der New Economy die Einkommensungleichheiten stetig verschärft und die Armut nicht nur zu wenig bekämpft, sondern noch vergrößert hat. Die Beweislast ist erdrückend. Die Weltbank schätzt, dass zurzeit $42 \%$ der Einwohner Indiens unter der globalen Armutsgrenze von 1,25 Dollar pro Tag leben - nach dem Zensus 2001 wären dies 456 Mio. Menschen! Drei von vier Indern, das heißt aktuell mehr als 800 Mio. Menschen, leben von weniger als zwei Dollar pro Tag (WoRld BANK 2008). Die indische Planungskommission geht von anderen Kriterien aus und kommt auf „nur“ $28,6 \%$. Der National Sample SuRvey (NSS 2007) weist nach, dass 57 Mio. Kinder unterernährt sind, davon $47 \%$ unter fünf Lebensjahren.

Im Problem der Armut kumulieren vielfältige Arten der Benachteiligung und Diskriminierung - aufgrund von Kaste, Klasse, Geschlecht, Ethnizität, Religion, Besitz etc. Trotz der erschütternden Zustände in den tausenden Slums der Megacities und Metropolen hat Armut überwiegend ein ländliches Gesicht. Die Achillesferse Landwirtschaft wurde bereits weiter oben charakterisiert.

Die extremste Manifestation von kontinuierlichem Hunger und ständiger Unterernährung ist das Ausmaß der Mortalität. Im Global Hunger Index (GHI) von 2008

\footnotetext{
9) Nachfolgende Aussagen stützen sich auf IIPS National Family Health Survey (2007), IFPRI Global Hunger Index (2009), IFPRI India State Hunger Index, die Essaysammlung von Utsa PatNAIK (2009), Arbeiten von Rahul Goswami (2009), Rainer Hörig (2010), Walter Fernandes (2010) und anderen.
} 
(International Food Policy Research Institute) findet sich Indien an 66. Stelle unter 88 untersuchten Nationen, im Jahr 2009 an 65. Stelle unter 84. Es bewegt sich damit ungefähr auf dem Niveau von Burkina Faso oder Zimbabwe. Dieser Index verbindet drei Dimensionen: (1) Anteil der Personen an der Bevölkerung, deren Kalorienaufnahme nicht ausreichend ist; (2) Anteil der untergewichtigen Kinder unter fünf Jahren; (3) Mortalitätsrate der unter 5-Jährigen. Der genau so konstruierte India StATE HungeR INDEX beweist, dass auch die ökonomisch bestgestellten Regionen trotz Urbanisierung und industrieller Entwicklung ein massives Hungerproblem haben. Es gibt keinen signifikanten Zusammenhang zwischen boomender Ökonomie und Reduzierung des Hungers (Maharashtra, Gujarat).

Landesweit hat die Verfügbarkeit von Nahrungsmitteln abgenommen. Die Einwohner des Bundesstaates Madhya Pradesh haben im Vergleich zu 1981 60\% weniger Getreide per capita zur Verfügung, und der Hungerindex vergibt dafür die Kategorie „extremely alarming“ im Ranking zwischen Tschad und Äthiopien. „Alarming“ erhalten die meisten anderen Bundesstaaten. Nur Kerala, Punjab und Andhra Pradesh fallen in die nächstbeste Kategorie „,serious“. Andhra Pradesh landet damit zwischen Sudan und Kenia, Westbengalen und Karnataka zwischen Malawi, Ruanda und Kambodscha. Die Kategorien „low“ und „moderate“ erreicht bisher keine Region Indiens! Zwölf der 17 erfassten Flächenstaaten Indiens mit einer Bevölkerungszahl von 885 Mio. (2008) fallen in das unterste Drittel des Global Hunger Index (InTERnATIONAL Food Policy Research Institute). Obwohl sich die Nahrungsmittelproduktion seit der Unabhängigkeit mehr als vervierfacht hat, nimmt ein Drittel der Gesamtbevölkerung heute nur 1.700 Kalorien pro Kopf zu sich. Geht man von der offiziell festgelegten Armutsgrenze von zumindest 2.400 Kalorien aus, fallen über $70 \%$ der Bevölkerung darunter. Indiens berühmter Nobelpreisträger Amartya SEN weist schon seit Jahrzehnten nach, dass Armut in diesem Land kein Produktions- sondern ein (sozioökonomisches) Verteilungsproblem ist. Trotzdem ist das vielfältig geförderte Agrobusiness nach wie vor Exporteur von Weizen und Reis.

Zum Hunger gesellen sich viele andere Komponenten: unzureichende medizinische Versorgung, hohe Kinder- und Säuglingssterblichkeit, damit auch geringere Lebenserwartung. 72 von 1.000 Lebendgeborenen sterben vor Erreichung des fünften Lebensjahres. Uttar Pradesh mit 550 von 1.000 steht gerade noch vor dem Sudan (UNICEF 2007).

\subsection{Verdrängungsprozesse - Adivasi, Dalits, Other Backward Classes (OBCs)}

Verdrängungen im ländlichen Raum und Zwangsumsiedlungen im Namen von „Entwicklung“ verschärfen die Hungerproblematik. Fernandes (2010, S. 24) schätzt die Zahl der Vertriebenen (Displaced Persons, DP) und Zwangsumgesiedelten (Projectaffected Persons, PAP) für den Zeitraum 1947-2000 auf 60 Mio., wobei rund 25 Mio. Hektar Land, mehr als die Hälfte davon Gemeinschaftsland (Common Property Resources, CPR) requiriert wurden, um Staudämme, Kraftwerke, Sonderwirtschaftszonen usw. anzulegen. Dies sind viermal so viele Menschen wie bei der Zwangsteilung Indiens und Pakistans „ausgetauscht“ wurden. Rund 40\% der Vertriebenen gehören 
den Adivasi (Stammesleuten, Ureinwohnern) an, die über $8 \%$ der Gesamteinwohner ausmachen, also ca. 100 Millionen. 20\% sind Dalits (Unberührbare), weitere 20\% stammen aus Other Backward Classes (OBCs) wie Fischer, Arbeiter in Steinbrüchen, Kleinstbauern. Waren Staudämme und Kraftwerksprojekte über Jahrzehnte die „Tempel des modernen Indien" (Pandit NeHRU), so zeigt die Planung und Einrichtung hunderter Special Economic Zones (SEZ) die Handschrift der NEP und das Aufholbedürfnis gegenüber China.

Adivasi werden vor allem vertrieben, weil ihre Herkunftsregionen den Großteil an natürlichen Ressourcen bergen (Kohle, Eisen, Uran, Gold usw.). Stammesangehörige kannten traditionell nur Gemeinbesitz. Aus der Kolonialzeit herübergerettet wurden Gesetze, die diesen zum Eigentum des Staates erklärten (der deshalb „frei“ über die Nutzung verfügen darf). Seit Generationen dringen Höherkastige, Großgrundbesitzer, Landentwickler, staatliche Planer usw. in die Lebensräume der Adivasi ein. Umweltzerstörung in großem Stil ist die Folge: flächenhafte kommerzielle Abholzung, Überweidung, Vertreibungen laufen täglich ab. Entschädigungszahlungen werden mit allen Tricks umgangen oder minimiert. ${ }^{10)}$ Die Dalits sind überwiegend landlose Taglöhner. Da sie kein Land besitzen, müssen sie auch nicht entschädigt werden. Kleinstbauern verlieren in der Agrarkrise durch Überschuldung (Wucherzinsen) ihr letztes Stück Land. Von der Verarmung zur sozialen Ausgrenzung ist es nur noch ein Schritt.

Kurzum - Indiens Arme zahlen bisher den Preis für die Entwicklung des Landes. Im Human Development Index (HDI) des United Nations Development Programme (UNDP) von 2009 liegt Indien an 134. Stelle unter 182 Nationen (UNDP 2009). Weltmacht Indien?

Es gibt aber auch Zeichen der Hoffnung. Seit 2006 existiert das Mahatma Gandhi National Rural Employment Guarantee Scheme (MGNREGS), inzwischen landesweit eingesetzt. Es garantiert jedem ländlichen Haushalt 100 Tage Beschäftigung im Jahr mit einem festgesetzten Mindestlohn zum Aufbau der ländlichen Infrastruktur (80 Rupien oder 1,5 Euro pro Tag). Umfang und Reichweite dieses Programms zur Bekämpfung der Armut sind weltweit einzigartig. Im Haushaltsjahr 2009/10 waren dafür 6,2 Mrd. Euro veranschlagt und 45 Mio. haben tatsächlich Arbeit bekommen. Adivasi und Dalits stellen 57\% aller MGNREGS-Arbeiter und 42\% der Begünstigten sind Frauen (für die Mehrheit die erste Chance auf ein Einkommen). Das verbriefte Recht auf Arbeit, Schulbildung (seit 2002) und hoffentlich bald auch auf Nahrung ist ein echter Strategiewechsel weg von teuren Subventionen und korrupten Förderprogrammen. Amartya SEN darf es noch erleben - Hilfe zur Selbsthilfe (,empowerment“) und Rechtsanspruch auf Unterstützung (,entitlement“). Seit 2007 existiert der Right to Information Act (RTI), der jede Behörde zwingt, alle Anfragen von Privatpersonen innerhalb von 30 Tagen zu beantworten. Er erweist sich als gute Waffe gegen die überbordende Korruption auf den lokalen und regionalen Verwaltungsebenen.

\footnotetext{
10) Vgl. Adivasi-Rundbrief 37, 2010, beigegeben SüDASIEn 1/2010. „Wir brauchen Essen, nicht Stahl“. Die Aktivistin Dayamani Barla kämpft gegen die Vertreibung von 70.000 Menschen aus rund 40 AdivasiDörfern durch den Giganten Arcelor/Mittal, der in Jharkhand für $8 \mathrm{Mrd}$. Dollar ein Stahlwerk mit einer Kapazität von 12 Mio. t. jährlich errichten will.
} 
HeINZ NiSSEL

\subsection{Widerstand}

Nicht alle Unterdrückten Indiens reagieren weiterhin gottergeben auf die Diskriminierungen. Während Umweltaktivisten seit Jahrzehnten friedliche Wege der Konfliktbeilegung bevorzugten (etwa das Chipko Movement zur Rettung der Wälder oder der Kampf gegen die Narmada-Staudämme), droht nun ein Flächenbrand im Hungergürtel Indiens. Dieser sogenannte ,rote Korridor" reicht von Bihar und Westbengalen über Jharkhand, Chattisgarh, Madhya Pradesh, Orissa bis in den südlichen Dekkan. Die neue Unionsregierung geht seit ihrem Amtsantritt Mitte Mai 2009 entschlossen gegen die Maoisten vor, und damit haben sich die Auseinandersetzungen zu einem regelrechten Bürgerkrieg ausgeweitet.

Ursprung und Name der maoistischen Bewegung der Naxaliten gehen auf einen Aufstand von Bauern gegen Großgrundbesitzer unter Führung Intellektueller der Universität von Kalkutta zurück, der im Jahr 1967 im westbengalischen Dorf Naxalbari begann. Der Aufstand wurde damals niedergeschlagen, aber die neo-marxistische Ideologie lebte immer fort und fand in der zunehmenden Ungleichheit des neoliberalen Wirtschaftens neue Nahrung. Zwischen 2001 und 2009 haben die Naxaliten ihre Präsenz von 56 auf 223 Distrikte ausgeweitet. Damit sind sie in mehr als der Hälfte der Bundesstaaten und auf nahezu einem Drittel des Staatsgebiets aktiv. Ihr gut organisierter harter Kern wird auf 10.000 bis 20.000 „Kämpfer“ geschätzt. Besonders stark ist der Einfluss der Maoisten in Gebieten hoher Adivasi-Anteile. Massaker an Polizisten und Parteifunktionären sowie das Herbeiführen von Zugentgleisungen gehören zu den spektakulären Aktionen. In den „befreiten Zonen“ hingegen erhalten die Einwohner Gratifikationen, werden aber auch in die Guerilla-Gruppen gepresst.

Leider will die Regierung nicht die sozioökonomischen Ursachen des Konflikts beseitigen, sondern setzt zur militärischen Gegenoffensive an. Schon im April 2006 bezeichnete Premier Manmohan SiNGH die Naxaliten als ,größte innenpolitische Herausforderung, die sich unserem Land jemals gestellt hat". Seit November 2009 läuft die groß angelegte Operation Green Hunt. Geplant ist der Einsatz von bis zu 75.000 Mann durch fünf Jahre mit riesigen Zwangsumsiedlungen der Stammesleute in Regierungscamps. Die Herstellung von ,law and order" ist hoffnungslos, so lange nicht dem eigentlichen Nährboden - der permanenten Vernichtung des Lebensraumes der Adivasi - Einhalt geboten wird.

\section{Fazit und Ausblick}

Was definiert eine Weltmacht und wie unterscheidet sich eine zukünftige Weltmacht von einer traditionellen? Traditionelle Mächte hielten eine Weltordnung im Lot, wie das British Empire in seiner Glanzzeit oder die Supermächte USA und Sowjetunion in Zeiten des Kalten Krieges. Die unipolare Phase der „Pax Americana“ seit 1990 geht zu Ende. Wie wird die Zukunft aussehen? Als wahrscheinlich erscheint aus heutiger Sicht eine multipolare Weltordnung - die USA, China als künftige Supermächte, dann auch Indien, Russland, die Europäische Union und Brasilien als Weltmächte. Oder entwi- 
ckelt sich eine neue internationale Ordnung, die gar keine Weltmächte mehr zulässt, weil die Macht der Nationalstaaten in entgrenzten Räumen zunehmend entschwindet und globalen bis lokalen Akteuren Platz macht? Oder muss man mit Saskia SASSEN die These vertreten (2008), dass Globalisierung innerhalb des Nationalen stattfindet und dort die Neuverteilung von Macht prägt?

Als unleugbar erscheint jedenfalls die Verschiebung des Gravitationszentrums der globalen Einflusskräfte im 21. Jh. nach Asien, nach China, Indien und Japan. Im Selbstverständnis dieser Nationen handelt es sich um ein Wiedererstarken der größten und ältesten Hochkulturen der Erde nach einer Schwächephase kolonialer Demütigungen. Die Bertelsmann-Stiftung hat 2007 mehr als 10.000 Menschen weltweit befragt, wer die zukünftigen Weltmächte sein werden. Wirtschaftliche Macht, politische Stabilität und einen leistungsfähigen Bildungs- und Forschungssektor nannten die Hälfte der Probanden als Kernelemente zukünftiger Macht; deutlich weniger militärische Macht, kulturelle Anziehungskraft oder Rohstoffreichtum. Trotz eines gestiegenen Bewusstseins für die transnationalen Verflechtungen und globalen Herausforderungen dominieren in den Antworten immer noch nationale Sichtweisen und die Betonung nationaler Stärke zur Lösung von Problemen.

Aus Sicht der Bevölkerungen haben (künftige) Weltmächte drei wesentliche Aufgaben zu erfüllen: Bekämpfung der Armut, Erhalt der Umwelt und Einsatz für Demokratie und Menschenrechte. Es zeigen sich deutliche nationale Unterschiede in der Prioritätensetzung. Unangefochten werden die USA als Weltmacht gesehen (von $81 \%$ ), mit deutlichem Abstand, vor allem für die Zukunft, China (50\%). Nur (noch) die Europäer selbst betrachten die Europäische Union (EU) in Gegenwart und Zukunft als Weltmacht (34\%). Weit abgeschlagen liegt noch Indien mit $15 \%$ weltweiter Zustimmung, doch strotzen die indischen Befragten vor Selbstbewusstsein: $38 \%$ sind überzeugt von der Weltmachtrolle Indiens. Dies signalisiert ein deutlich erstarktes Selbstbild und Vertrauen in die eigenen Fähigkeiten.

Unabhängig von Befragungen und Ranglisten lässt sich ablesen, dass klassische Attribute des Charakters der Macht heute ausgedient haben und weltweite Legitimität anders „erworben“ werden muss. Noch ist Amerika die Supermacht schlechthin in allen Dimensionen, nicht nur im weltweiten Handeln an sich, sondern auch in der Gestaltung der Spielregeln, nach denen alle anderen Akteure zu handeln haben. Die Ingredienzien zukünftiger Weltmächte werden sich noch vielschichtiger entwickeln: enorme Wirtschaftskraft, große Bevölkerungszahl, hohes Bildungsniveau, militärische Fähigkeit zur Abschreckung, ein attraktives Wertesystem und ein handlungsfähiges politisches System, Befähigung zum regionalen Hegemon im unmittelbaren Umfeld, Anerkennung und Mit-Steuerung globaler Ordnungsrahmen (z.B. Menschrechte, Terrorismusbekämpfung), Befähigung zum Engagement für Ziele der Weltpolitik (z.B. UNO-Schutztruppen) - also verstärkt Elemente von „soft power“ - Fähigkeiten, die zum Frieden und zur gemeinsamen Sicherheit in der Welt beitragen. Die Gewichtung dieser Faktoren hängt von den internen Entwicklungen der Mächte wie von ihrem internationalen Agieren in den kommenden Jahren oder Jahrzehnten ab. Indien könnte eines Tages diese Bedingungen erfüllen. Aber wann?

Immer wieder im Fokus befinden sich auch die BRIC-Staaten (Brasilien, Russland, Indien, China), die als große Aufsteiger („top emerging economies“) miteinander die 
Welt verändern könnten. Sie umfassen $40 \%$ der Menschheit, verwalten schon heute $20 \%$ des Gesamteinkommens, halten $40 \%$ der Devisenreserven, gehören zu den größten Konsumenten und Rohstoffproduzenten. Aber alle sind sie nationale Akteure mit je eigenen Entwicklungszielen und -strategien, sehr unterschiedlichen Interessen und wenig Gemeinsamkeiten. Sie stimmen (bisher) nur in wenigen Positionen überein.

Dass Indien beständig an Bedeutung gewinnt, steht heute außer Zweifel. Doch wurden internationale Beobachter nicht selten geblendet von den phänomenalen wirtschaftlichen Erfolgen in der letzten Dekade. Und desgleichen finden vollmundige Prognosen über die glänzende Zukunft Indiens innerhalb des Landes ein begeistertes Echo. ,Schon seit der Staatsgründung waren die internationalen Ambitionen der Inder immer bemerkenswert unbescheiden“ (KHILNANI 2010, S. 11), getragen vom Bewusstsein der kulturellen Überlegenheit (gegenüber dem Westen) und einer gewaltlosen Politik mit hohen moralischen Prinzipien. GandHIs Vision, dass scheinbare Schwäche sich in Stärke verwandeln könne, transformierte NEHRU zu einem politischen Gestaltungsprinzip: Indien als legitimierter Führer der Schwachen in der Welt (damals die Bewegung der Blockfreien). Die Definition von der „größten Demokratie der Welt“ erfüllt die Inder mit Stolz. Zunehmend ängstlich blickt Europa nach Osten, riegelt sich protektionistisch ab (Ökonomie) oder wirkt zunehmend orientierungslos (Politik). Für den Westen ist dieses demokratische Indien ein Gegenentwurf zu China, oder jedenfalls der ,,angenehmere Geschäftspartner“.

In jüngster Zeit mehren sich wiederum die Stimmen, die Indien die Fähigkeit absprechen eine Weltmacht zu sein oder zu werden. Le Monde DIPLOMATIQUE betitelt ihre Sonderausgabe 2010 lakonisch „Indien, die barfüßige Großmacht“. GuHA (2010) spricht von „Großmachtphantasien. Warum Indien in naher Zukunft nicht zur Großmacht aufsteigen kann“. Und KHILNANi (2010) meint, eine Supermacht werde Indien so schnell nicht werden, aber ein Vermittler und Brückenbauer, ein ungewöhnlicher globaler Akteur, nämlich ein wichtiger, ernstzunehmender Staat mit einer armen Bevölkerung. (Bisher galt die Gleichsetzung von armer Bevölkerung mit politischer Einflusslosigkeit.) Von der Handelspolitik bis zur Klimapolitik könnte Indien zu einem Sprachrohr für die ,underdogs“ dieser Welt werden, als Vermittler zwischen USA und China, zwischen G-20 und G-77. Eine zu optimistische Einschätzung?

Vielfältig sind die Gründe, die Indiens Aufstieg hemmen (können). Seine geostrategische Lage in Südasien ist denkbar ungünstig. Die beiden unmittelbaren Rivalen des ungeliebten Hegemon in Südasien - China und Pakistan - verfügen über Atomwaffen und riesige konventionelle Streitkräfte. Zu allen Nachbarn sind die Beziehungen angespannt bis bestenfalls ,normalisiert“, sichere Grenzen können nur mit enormem militärischen Aufwand erkauft werden. China hat bisher im „rat race“ um Einfluss und Ressourcen weltweit die Nase vorn.

Vielfältig bleiben die Spaltungen im Land selbst. Die sozialen Spannungen sind beträchtlich, die Kluft zwischen Arm und Reich hat sich seit dem neoliberalen Wirtschaftskurs nochmals stark vergrößert. Die Religionskonflikte, vor allem zwischen Hindus und Moslems, sind virulent und jederzeit gefährlich, Kastenegoismen zersetzen staatliche Institutionen, der Kampf der Dalits und Adivasi, die rapide Zerstörung der Umwelt, die Krise der Landwirtschaft, Aufstände der Minoritäten im Nordosten, versagende Primärerziehung, Kinderarbeit ... die Liste erscheint endlos. Was bleibt, 
ist ein Bild voller Widersprüche. Stabilisierte Ungleichheit und Ungleichzeitigkeit in allen Dimensionen. Die an den Anfang dieses Beitrags gestellten Leitmotive „stimmen“.

Weltmacht Indien - Realität oder Chimäre? Eine abschließende persönliche Einschätzung: Langfristig ist eine Positionierung Indiens als „sanfte Weltmacht“ im neuen Politikverständnis möglich, wenn die wichtigsten Grundbedingungen dafür geschaffen werden. Dazu zählen im Inneren die energische Bekämpfung der Armut und nicht der Armen auf allen Ebenen, die Bewahrung des säkularen Staates mit seiner demokratischen Verfasstheit, die Zurückdrängung des religiösen und politischen Extremismus; in der Außenpolitik die Aussöhnung und Friedenssicherung mit seinen unmittelbaren Nachbarn in Südasien sowie global als demokratisches Flaggschiff im Spiel der Mächtigen als ernst genommener Vermittler zwischen Erster und Dritter Welt.

\section{Literaturverzeichnis}

Ahuja R., Brosius Ch. (Hrsg.) (2006), Mumbai - Delhi - Kolkata. Annäherungen an die Megastädte Indiens. Heidelberg, Draupadi Verlag.

Amin S. (2007), Weltmacht Indien? Der Subkontinent zwischen kolonialem Erbe und globalem Aufstieg. In: Blätter f. dt. u. intern. Politik, 6, S. 705-716.

AsienKurier Nr. 38, 7/2010 - http://pdf.asienkurier.com

Bertelsmann-STiftung (Hrsg.) (2007), Wer regiert die Welt? Ergebnisse der zweiten Repräsentativbefragung in den Ländern Brasilien, China, Deutschland, Frankreich, Großbritannien, Indien, Japan, Russland und USA. Oktober 2007. - http://www.bertelsmann-stiftung.de

BRonger D., W AMSER J. (2005), Indien - China. Vergleich zweier Entwicklungswege. Münster, LIT Verlag.

Bundeszentrale für politische Bildung (Hrsg.) (2007), Indien (= Informationen z. polit. Bildung, 296). Bonn.

Das G. (2006), The India Model. In: Foreign Affairs, 85/4, S. 2-16.

Deutsche Bank (DB) Research (Hrsg.) (2005), Outsourcing nach Indien: der Tiger auf dem Sprung. - http://www.dbresearch.de

Deutsche Bank (DB) Research (Hrsg.) (2006), Indien - Auf dem Weg zur Weltmacht? - http:// www.dbresearch.de

Deutsche Bank (DB) Research (Hrsg.) (2008), Infrastruktur Indien: 450 Mrd. Gründe, jetzt zu investieren. - http://www.dbresearch.de

Deutsche Gesellschaft für Auswärtige Politik (DGAP) (Hrsg.) (2006), Zeitschrift Internationale Politik (IP), 61, 10: Weltmacht Indien.

Edition Le Monde diplomatique No. 7 (2010), Indien. Die barfüßige Großmacht. Dt. Ausg. Berlin, taz Verlag.

Еммотт B. (2009), Rivals. How the Power Struggle between China, India and Japan will shape our next decade. Brighton, Penguin, Penn Press Publ.

Engelmeier T. (2009), Nation-Building and Foreign Policy in India. New Delhi, Cambridge Univ. Press India.

Fernandes W. (2010), Wer zahlt den Preis für den Fortschritt? Die industrielle Entwicklung und ihre Opfer. In: Südasien, 1, S. 24-28.

Fritz E. (Hrsg.) (2010), Entwicklungsland, Schwellenland, Global Player: Indiens Weg in die Verantwortung. Oberhausen, Athena-Verlag. 
Ganguly S. (2010), India‘s Foreign Policy - Retrospect and Prospect. New Delhi, Oxford Univ. Press India.

Ghosh J. (2010), Die verdeckte Finanzkrise. In: Le Monde Diplomatique, 7, S. 40-45.

Goldman \& SAChs (Hrsg.) (2003), Global Economics Paper No. 99: Dreaming with BRICs: The Path to 2050. - http://www2.goldmansachs.com/insight/research/reports/report6.html

Goswami R. (2009), How Distant a Square Meal: The Persistence of Hunger in India. In: Infochange India, Jan 11-17, 2009. - http://infochangeindia.org/200901097562/

Government of India (GOI) (Hrsg.), Census of India. - http://www.censusindia.net oder http:// www.censusindia.gov.in

Government of India (GOI) (Hrsg.) (2008), Prime Minister's Council on Climate Change: National Action Plan on Climate Change. 30. Juni 2008.

Hansen S. (2010), Jetzt sind wir dran. In: Le Monde Diplomatique, 7, S. 50-54.

Hauff M. von (Hrsg.) (2009), Indien. Herausforderungen und Perspektiven. Marburg, Metropolis Verlag.

HimmelReich J. (2010), Villa Kunterbunt. Wer Indien verstehen will, muss lesen. Viel lesen. http://www.internationalepolitik.de/buecherschau/view/1277801382.html

HörIG R. (2010), 100 Tage Arbeit. In: Le Monde Diplomatique, 7, S. 14-17.

Ihlau O. (2008), Weltmacht Indien. Die neue Herausforderung des Westens. München, Pantheon Verlag (München, Siedler Verlag 2006).

Imhasly B. (2006), Abschied von Gandhi. Eine Reise durch das neue Indien. Freiburg, Verlag Herder.

India State Hunger INDEX (IFPRI) - http://www.ifpri.org/pubs/cp/ishio8.asp

Indian Space Research Organisation (ISRO) (Hrsg.) (laufend) - http://www.isro.org

International Food Policy Research Institute (IFPRI) (Hrsg.), Global Hunger Index 2008. http://www.ifpri.org/pubs/cp/GHIO8.asp

International Institute for Population Sciences (IIPS) (Mumbai), Macro International (Hrsg.) (9/2007), Third National Family Health Survey, Summary of Findings.

International Institute For Strategic Studies (IISS) (Hrsg.) (2010), laufende Untersuchungen. - http://www.iiss.org/publications/military-balancel

Jha P., Negre M. (2007), Der Preis des Wunders - Indien zwischen wirtschaftlichem Aufstieg und sozialem Abstieg. In: Blätter f. dt. u. intern. Politik, 10, S. 1245-1256.

Kapur P., Ganguly S. (2010), India, Pakistan, and the Bomb: Debating Nuclear Stability in South Asia. New York, Columbia Univ. Press.

Khilnani S. (2010), Die sanfte Brückenmacht. In: Intern. Politik, Juli/August, S. 10-19.

Kugler T., Swaminathan S. (2006), The politics of population. In: Intern. Studies Review, 8, S. 581-596.

LucE E. (2007), In Spite of the Gods. The Strange Rise of Modern India. New York, Doubleday.

Lutz W., Scherbov S. (2005), India's window of opportunity (= Options Magazine, Autumn 2005), Laxenburg, Intern. Institute for Applied Systems Analysis (IIASA).

Mann M. (2010), Geschichte Südasiens 1500 bis heute. Darmstadt, Wiss. Buchges.

Müller H. (2006, 2007²), Weltmacht Indien. Wie uns der rasante Aufstieg herausfordert. Frankfurt am Main, Fischer TB

Müller H., Rauch C. (2008), Indiens Weg zur Wirtschaftsmacht. In: Aus Politik und Zeitgeschichte (APuZ), 22, Beilage zu „Das Parlament“.

Müller O. (2006), Wirtschaftsmacht Indien. Chance und Herausforderung für uns. München, Carl Hanser Verlag.

National Association of Software and Services Companies (NASSCOM) (Hrsg.) (2010), NASSCOM Strategic Review 2010, NASSCOM Perspective 2020 Executive Summary. - http://www.nasscom.in 
Nissel H. (2006), Bombay/Mumbai: Stadtumbau und Stadterweiterung einer „Globalizing City“. In: Ahuja R., Brosius Ch. (Hrsg.), Mumbai - Delhi - Kolkata. Annäherungen an die Megastädte Indiens, S. 19-34. Heidelberg, Draupadi Verlag.

Nissel H. (2007), Der Kampf um Lebensraum in den Megastädten Indiens. In: Geogr. Jahresbericht aus Österreich, LXII und LXIII, S. 131-155.

Nissel H. (2007), Indien. In: LandesverteidigungsaKademie (Hrsg.), Aspekte zur Vision BH 2025 (= Schriftenreihe d. Landesverteidigungsakademie, 7/2007), S. 299-315. Wien.

Nissel H. (2008), Indien - Globaler und regionaler geopolitischer Akteur? In: RiEMER A.K. (Hrsg.), Geopolitik großer Mächte: Aktuelle Trends (= Schriftenreihe d. Landesverteidigungsakademie, 4/2008), S. 205-241.

Nissel H. (2009), Materialien zur Vorlesung Südasien I, WS 2008/09, Univ. Wien.

Nissel H. (2009), Contesting Urban Space: Megacities and Globalization in India. In: Geogr. Rundschau, Intern. Ed., 5, 1, S. 40-46.

Nissel H. (2009), Indien hat gewählt. Ergebnis und Bewertung der 15. Unterhauswahlen im April/Mai 2009 (= ISS Flash Analysis 5). Wien, Inst. f. Strategie u. Sicherheitspolitik d. Landesverteidigungsakademie Wien. 5 S. (registrierter online-Zugang).

Panagariya A. (2008), India. The Emerging Giant. Oxford Univ. Press USA.

PatNaik U. (2009), Unbequeme Wahrheiten. Hunger und Armut in Indien. Heidelberg, Draupadi Verlag.

Pilny K. (2006), Tanz der Riesen. Indien und China prägen die Welt. Frankfurt am Main - New York, Campus Verlag.

Rothermund D. (2008), Indien. Aufstieg einer asiatischen Weltmacht. München, Verlag C.H. Beck.

SASSEN S. (2008), Das Paradox des Nationalen. Territorium, Autorität und Rechte im globalen Zeitalter. Frankfurt am Main, Verlag Suhrkamp.

Schoеtтl U. (2009), Indien. Profil einer neuen Grossmacht. Zürich, Verlag Neue Zürcher Zeitung.

Six C. (2007), Hindi - Hindu - Hindustan: Politik und Religion im modernen Indien. Wien, Mandelbaum Verlag.

Stockholm International Peace Research Institute (SIPRI) (Hrsg.), SIPRI Yearbook 2010 und frühere. - http://www.sipri.org/yearbook

SüDAsiEn, Zeitschrift des Südasienbüros, 30, 1/2010. Bonn.

Thakur R. (2006), Der Elefant ist aufgewacht. In: Intern. Politik, 10, S. 6-12.

Tharoor Sh. (2006), Das Immer-und-ewig-Land. In: Intern. Politik 10, S. 46-50.

Transparency International (Hrsg.) (2009), CPI, Corruption Perception Index (Public Sector Corruption). - http://www.transparency.org/policy.research/cpi/2009)

W AGner Ch. (2006), Das politische System Indiens. Eine Einführung. Wiesbaden, Vs.

Winters A.L., Shahid Y. (Hrsg.) (2007), Dancing with Giants - China, India, and the Global Economy. Washington D.C., World Bank.

United Nations (Hrsg.) (jährlich), Human Development Report.

United Nations Development Programme (UNDP), Human Development Reports (HDR) seit 1990. - http://hdrundp.org/ und http://www.undp.org/

United Nations International Children's Emergency Fund (UNICEF) 2007 - http://www. unicef.org/

World B ANK (Hrsg.) (jährlich), World Development Report. - http://siteresources.worldbank. org/INTWDR/

ZiNGEL W.-P. (2009), Wirtschaft und Umwelt - Indische Lösungsansätze zu einem nicht nur indischen Problem. In: von Hauff M. (Hrsg.), Indien. Herausforderungen und Perspektiven, S.133-155. Marburg, Metropolis Verlag.

Anmerkung: alle Links wurden zuletzt im Juli 2010 überprüft. 
Wichtige Tageszeitungen und Journale (in Auswahl):

The Times of India - http://timesofindia.indiatimes/com

Indian Express - http://expressbuzz.com/

The Hindu - http://www.hinduonnet.com/

India Today - http://indiatoday.intoday.in/site/

The Economic Times - http://economictimes.indiatimes.com

Frontline - http://www.flonnet.com/

Economic and Political Weekly (EPW) - http://epw.in/epw/userindex.jsp 